\title{
FIC/FEM formulation with matrix stabilizing terms for incompressible flows at low and high Reynolds numbers
}

Received: 19 December 2005 / Accepted: 14 February 2006

(C) Springer-Verlag 2006

\begin{abstract}
We present a general formulation for incompressible fluid flow analysis using the finite element method. The necessary stabilization for dealing with convective effects and the incompressibility condition are introduced via the Finite Calculus method using a matrix form of the stabilization parameters. This allows to model a wide range of fluid flow problems for low and high Reynolds numbers flows without introducing a turbulence model. Examples of application to the analysis of incompressible flows with moderate and large Reynolds numbers are presented.
\end{abstract}

Keywords Stabilized formulation - Incompressible fluid . Finite calculus · Finite element method · High Reynolds numbers . Turbulence model

\section{Introduction}

Much effort has been spent in developing the so called stabilized numerical methods overcoming the two main sources of instability in incompressible flow analysis, namely those originated by the high values of the convective terms and those induced by the difficulty in satisfying the incompressibility condition.

The solution of above problems in the context of the finite element method (FEM) has been attempted in a number of ways. The first attempts to correct the underdiffusive character of the Galerkin FEM for high convection flows were based in adding some kind of artificial viscosity terms to the standard Galerkin equations $[1,2]$.

A popular way to overcome the problems with the incompressibility constraint is by introducing a pseudo-compressibility in the flow and using implicit and explicit algorithms developed for this kind of problems such as artificial compressibility schemes [3-5] and preconditioning techniques

E. Oñate $(\bowtie) \cdot$ A. Valls $\cdot$ J. García

International Center for Numerical Methods in Engineering,

Universidad Politécnica de Cataluña,

Gran Capitán s/n, 08034 Barcelona, Spain,

E-mail: onate@cimne.upc.edu

URL: www.cimne.upc.es
[6]. State of the art FEM schemes for fluid flow analysis with good stabilization properties for the convective and incompressibility terms are based in Petrov-Galerkin (PG) techniques. The background of PG methods are the noncentred (upwind) schemes for computing the first derivatives of the convective operator in finite difference and finite volume methods [7, 8]. A general class of stabilized PG FEM has been recently developed where the standard Galerkin variational form of the momentum and mass balance equations is extended with adequate residual-based terms in order to achieve a stabilized numerical scheme. References [9-30] list some of the more popular stabilized FEM of this kind. A review of many of these methods can be found in [1, 31].

In this paper a stabilized FEM for incompressible flows is derived via a finite calculus (FIC) approach [32, 33]. The FIC method is based in invoking the balance of fluxes in a fluid domain of finite size. This introduces naturally additional terms in the classical differential equations of momentum and mass balance of infinitesimal fluid mechanics which are a function of characteristic length dimensions related to the element size in the discretized problem. The FIC terms in the modified governing equations provide the necessary stabilization to the discrete equations obtained via the standard Galerkin FEM. The FIC/FEM formulation allows to use low order finite elements (such as linear triangles and tetrahedra) with equal order approximations for the velocity and pressure variables.

The FIC/FEM formulation has proven to be very effective for the solution of a wide class of problems, such as convection-diffusion [32-39] and convection-diffusion-reaction [40-42] involving arbitrary high gradients, incompressible flow problems accounting for free surface effects and fluidstructure interaction situations [32, 33, 43-51] and quasi and fully incompressible problems in solid mechanics $[52,53]$.

The FIC equations for incompressible flow derived in previous works of the authors assumed that the dimensions of the domain where the momentum conservations law was enforced remain the same independently of the direction along which balance of momentum is imposed. As a consequence, 
each of the resulting FIC momentum equations contain the same characteristic dimensions which can be grouped in a characteristic distance vector. In this paper, a refined FIC momentum equations are derived by accepting that the dimensions of the momentum balance domain are different for each of the momentum equations. This introduces a matrix form of the characteristic distances and of the corresponding FIC terms which have better intrinsic stabilization properties.

The idea of a matrix form of the stabilization parameters is close to the element-matrix-based and element-vector-based stabilization parameters proposed in [54] where different intrinsic time parameters were defined separately for each degree of freedom of the equation system.

Stabilized FEM have been successfully used in the past to solve a wide range of fluid mechanics problems. The intrinsic dissipative properties of the stabilization terms (which can interpreted as an additional viscosity) typically suffices to yield good results for low and moderate values of the Reynolds number $(R e)$. For high values of $R e$ most stabilized FEM fail to provide physically meaningful results and the numerical solution is often unstable or inaccurate. The introduction of a turbulence model is mandatory in order to obtain meaningful results in these cases.

The relationship between the additional dissipation introduced by the turbulence model and the intrinsic dissipative properties of stabilized FEM is an open topic which is attracting increasing attention in the CFD community. It is clear that both remedies (the turbulence model and the stabilization terms) play a similar role in the numerical solution, i.e. that of ensuring a solution which is "physically sound" and as accurate as possible.

It is our belief that the matrix stabilization terms introduced by the FIC/FEM formulation here presented allow to model accurately high $R e$ number flows without the need of introducing any turbulence model. The background of this belief originates in the positive experiences in the application of a very similar formulation for solving advection-diffusion and advection-diffusion-reaction problems with arbitrary sharp gradients without introducing any transverse dissipation terms $[39,41]$. The extension of these ideas to the $\mathrm{Na}-$ vier-Stokes equations described here provides a straightforward procedure for solving a wide class of flow problems from low to high Reynolds numbers, as demonstrated by the good results presented in the paper.

The layout of the paper is the following. In the next section the FIC equations for incompressible flows with matrix stabilization terms are presented. The finite element discretization is introduced and the resulting matrix equations are detailed. A fractional step scheme for the transient solution is detailed. Examples of applications to the 2D analysis of flows passing a backward facing step and a cylinder at different Reynolds numbers are presented.

\section{General FIC equations for viscous incompressible flow}

The FIC governing equations for a viscous incompressible fluid can be written in an Eulerian frame of reference as

\section{Momentum}

$r_{m_{i}}-\frac{1}{2} h_{i j} \frac{\partial r_{m_{i}}}{\partial x_{j}}=0$ in $\Omega$ no sum in $i$.

Mass balance

$r_{d}-\frac{1}{2} h_{j} \frac{\partial r_{d}}{\partial x_{j}}=0$ in $\Omega$,

where

$r_{m_{i}}=\rho\left(\frac{\partial u_{i}}{\partial t}+u_{j} \frac{\partial u_{i}}{\partial x_{j}}\right)+\frac{\partial p}{\partial x_{i}}-\frac{\partial s_{i j}}{\partial x_{j}}-b_{i}$

$r_{d}=\frac{\partial u_{i}}{\partial x_{i}} \quad i, j=1, n_{d}$.

Above $\Omega$ is the analysis domain, $n_{d}$ is the number of space dimensions ( $n_{d}=2$ for $2 \mathrm{D}$ problems), $u_{i}$ is the velocity along the ith global axis, $\rho$ is the (constant) density of the fluid, $p$ is the absolute pressure (defined positive in compression), $b_{i}$ are the body forces and $s_{i j}$ are the viscous deviatoric stresses related to the viscosity $\mu$ by the standard expression

$s_{i j}=2 \mu\left(\dot{\varepsilon}_{i j}-\delta_{i j} \frac{1}{3} \frac{\partial u_{k}}{\partial x_{k}}\right)$,

where $\delta_{i j}$ is the Kronecker delta and the strain rates $\dot{\varepsilon}_{i j}$ are

$\dot{\varepsilon}_{i j}=\frac{1}{2}\left(\frac{\partial u_{i}}{\partial x_{j}}+\frac{\partial u_{j}}{\partial x_{i}}\right)$.

The FIC boundary conditions are

$$
\begin{aligned}
n_{j} \sigma_{i j}-t_{i}+\frac{\frac{1}{2} h_{i j} n_{j} r_{m_{i}}}{u_{j}-u_{j}^{p}}=0 \text { on } \Gamma_{t} \text { no sum in } i \\
\Gamma_{u}
\end{aligned}
$$

and the initial condition is $u_{j}=u_{j}^{0}$ for $t=t_{0}$.

Summation convention for repeated indices in products and derivatives is used unless otherwise specified.

In Eqs. (6a) and (6b) $t_{i}$ and $u_{j}^{p}$ are surface tractions and prescribed displacements on the boundaries $\Gamma_{t}$ and $\Gamma_{u}$, respectively, $n_{j}$ are the components of the unit normal vector to the boundary and $\sigma_{i j}$ are the total stresses given by $\sigma_{i j}=s_{i j}-\delta_{i j} p$.

The $h_{i j}$ and $h_{j}$ are characteristic distances of the domain where balance of momentum and mass is enforced. In Eqs. (1) and (2) these lengths define the domain where equilibrium of boundary tractions is established [32]. In the discretized problem the characteristic distances become of the order of the typical element dimensions. Note that by making these distances equal to zero the standard infinitessimal form of the fluid mechanics equations is recovered $[1,7,31]$.

Equations (1)-(6) are the starting point for deriving stabilized FEM for solving the incompressible Navier-Stokes equations. The underlined FIC terms in Eq. (1) are essential to overcome the numerical instabilities due to the convective terms in the momentum equations, whereas the underlined terms in Eq. (2) take care of the instabilities due to the incompressibility constraint. An interesting feature of the FIC formulation is that it allows to use equal order interpolation for the velocity and pressure variables [43-53]. 
Remark 1 In previous work of the authors the characteristic distances in the momentum equations had a vector form, i.e. the FIC momentum equations were written as

$r_{i}-\frac{1}{2} h_{j} \frac{\partial r_{i}}{\partial x_{j}}=0$

or

$r_{i}-\frac{1}{2} \mathbf{h}^{\mathrm{T}} \nabla r_{i}=0$

where (for 2D problems) $\mathbf{h}=\left[h_{1}, h_{2}\right]^{\mathrm{T}}$ is the characteristic length vector [32, 33, 43].

The difference of Eqs. (7) with Eq. (1) is that the characteristic distances have now a matrix form, i.e. the expanded form of the momentum equations (1) is (for 2D problems)

$r_{1}-\frac{1}{2}\left(h_{11} \frac{\partial r_{1}}{\partial x_{1}}+h_{12} \frac{\partial r_{1}}{\partial x_{2}}\right)=0$

$r_{2}-\frac{1}{2}\left(h_{21} \frac{\partial r_{2}}{\partial x_{1}}+h_{22} \frac{\partial r_{2}}{\partial x_{2}}\right)=0$.

The rationale of Eqs. (8) is briefly explained in the Appendix.

The matrix of stabilization parameters $\mathbf{S}$ is defined as (for 2D problems)

$\mathbf{S}=\left[\begin{array}{ll}h_{11} & h_{12} \\ h_{21} & h_{22}\end{array}\right]$.

Remark 2 Note that the characteristic distances in the FIC mass conservation equation (2) have a vector form. As mentioned above distances $h_{1}$ and $h_{2}$ in Eq. (2) (for 2D problems) denote the dimensions of the domain where balance of mass is globally enforced (see Appendix). This is a basic difference with the momentum equations where the momentum balance law is applied along each global coordinate direction.

\subsection{Stabilized integral forms}

From the momentum equations it can be obtained $[43,45]$

$\frac{\partial r_{d}}{\partial x_{i}} \simeq \frac{h_{i i}}{2 a_{i}} \frac{\partial r_{m_{i}}}{\partial x_{j}}$, no sum in $i$,

where

$a_{i}=\frac{2 \mu}{3}+\frac{\rho u_{i} h_{i i}}{2}$, no sum in $i$.

Substituting Eq. (10a) into Eq. (2) and retaining the terms involving the derivatives of $r_{m_{i}}$ with respect to $x_{i}$ only, leads to the following alternative expression for the stabilized mass balance equation

$r_{d}-\sum_{i=1}^{n_{d}} \tau_{i} \frac{\partial r_{m_{i}}}{\partial x_{i}}=0$

with

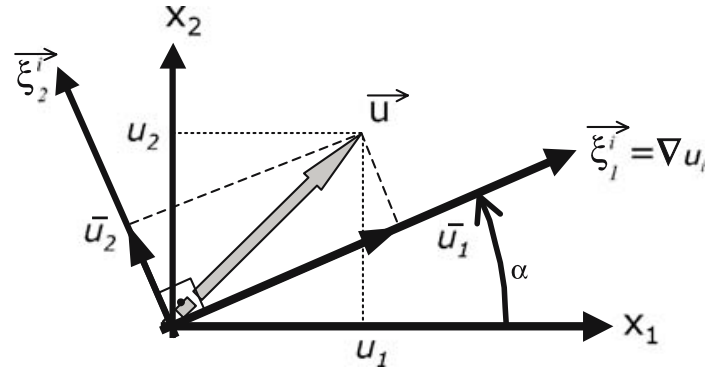

Fig. 1 Definition of the principal curvature direction $\vec{\xi}_{1}^{i}$ along the gradient of $u_{i}$

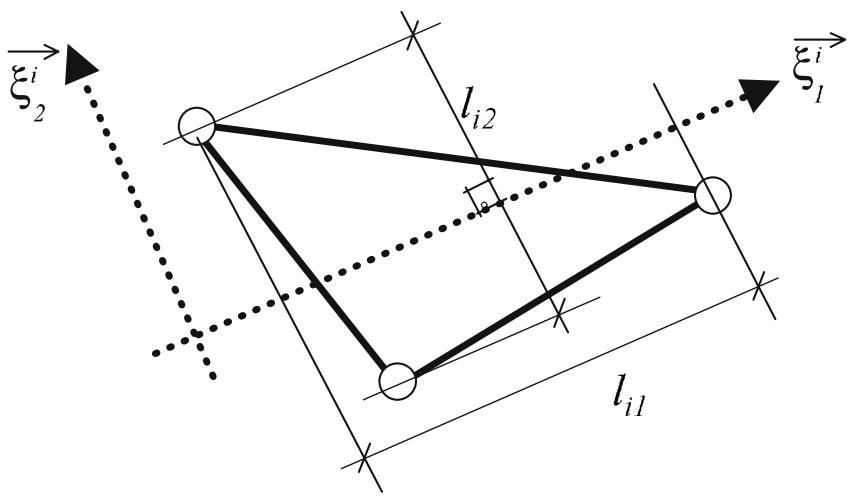

Fig. 2 Definition of the element characteristic distances $l_{i 1}$ and $l_{i 2}$ corresponding to the $i$ th momentum equation

$\tau_{i}=\left(\frac{8 \mu}{3 h_{i i} h_{i}}+\frac{2 \rho u_{i}}{h_{i i}}\right)^{-1}$

The $\tau_{i}$ 's in Eq. (11) when multiplied by the density are equivalent to the intrinsic time parameters, seen extensively in the stabilization literature. The interest of Eq. (11) is that it introduces the first space derivatives of the momentum equations into the mass balance equation. These terms have intrinsic good stability properties as explained next.

The weighted residual form of the momentum and mass balance equations [Eqs. (1), (11)] is written as

$$
\begin{aligned}
& \int_{\Omega} \delta u_{i}\left[r_{m_{i}}-\frac{h_{i j}}{2} \frac{\partial r_{m_{i}}}{\partial x_{j}}\right] \mathrm{d} \Omega \\
& +\int_{\Gamma_{t}} \delta u_{i}\left(\sigma_{i j} n_{j}-t_{i}+\frac{h_{i j}}{2} n_{j} r_{m_{i}}\right) \mathrm{d} \Gamma=0 \\
& \int_{\Omega} q\left[r_{d}-\sum_{i=1}^{n_{d}} \tau_{i} \frac{\partial r_{m_{i}}}{\partial x_{i}}\right] \mathrm{d} \Omega=0,
\end{aligned}
$$

where $\delta u_{i}$ and $q$ are arbitrary weighting functions representing virtual velocities and virtual pressure fields. Integrating by parts the $r_{m_{i}}$ terms in Eqs. (13) and (14) leads to 

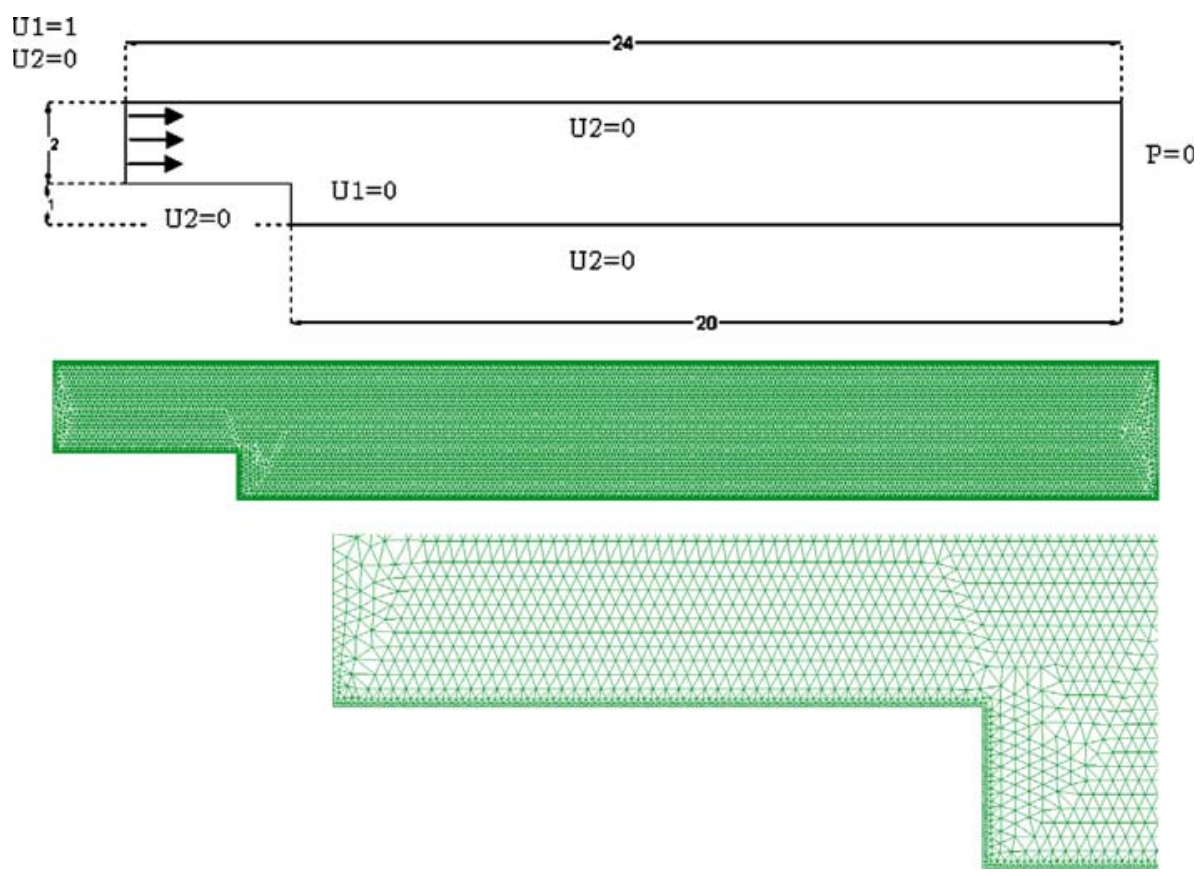

Fig. 3 Backward facing step. Geometry and finite element mesh of 30,850 three-noded triangles. Mesh detail at the vicinity of the step

$$
\begin{aligned}
& \int_{\Omega} \delta u_{i} r_{m_{i}} \mathrm{~d} \Omega+\int_{\Gamma_{t}} \delta u_{i}\left(\sigma_{i j} n_{j}-t_{i}\right) \mathrm{d} \Gamma \\
& +\int_{\Omega} \frac{h_{i j}}{2} \frac{\partial \delta u_{i}}{\partial x_{j}} r_{m_{i}} \mathrm{~d} \Omega=0 \\
& \int_{\Omega} q r_{d} \mathrm{~d} \Omega+\int_{\Omega}\left[\sum_{i=1}^{n_{d}} \tau_{i} \frac{\partial q}{\partial x_{i}} r_{m_{i}}\right] \mathrm{d} \Omega \\
& -\int_{\Gamma}\left[\sum_{i=1}^{n_{d}} q \tau_{i} n_{i} r_{m_{i}}\right] \mathrm{d} \Gamma=0 .
\end{aligned}
$$

We will neglect hereonwards the third integral in Eq. (15b) by assuming that $r_{m_{i}}$ is negligible on the boundaries. The deviatoric stresses and the pressure terms in the first integral of Eq. (15a) are integrated by parts in the usual manner. The resulting momentum and mass balance equations are

$$
\begin{gathered}
\int_{\Omega}\left[\delta u_{i} \rho\left(\frac{\partial u_{i}}{\partial t}+u_{j} \frac{\partial u_{i}}{\partial x_{j}}\right)+\frac{\partial \delta u_{i}}{\partial x_{j}}\left(\mu \frac{\partial u_{i}}{\partial x_{j}}-\delta_{i j} p\right)\right] \mathrm{d} \Omega \\
\quad-\int_{\Omega} \delta u_{i} b_{i} \mathrm{~d} \Omega-\int_{\Gamma_{t}} \delta u_{i} t_{i} \mathrm{~d} \Gamma \\
\quad+\int_{\Omega} \frac{h_{i j}}{2} \frac{\partial \delta u_{i}}{\partial x_{j}} r_{m_{i}} \mathrm{~d} \Omega=0 \\
\int_{\Omega} q \frac{\partial u_{i}}{\partial x_{i}} \mathrm{~d} \Omega+\int_{\Omega}\left[\sum_{i=1}^{n_{d}} \tau_{i} \frac{\partial q}{\partial x_{i}} r_{m_{i}}\right] \mathrm{d} \Omega=0 .
\end{gathered}
$$

In the derivation of the viscous term in Eq. (16a) we have used the following identity holding for incompressible fluids (prior to the integration by parts)

$\frac{\partial s_{i j}}{\partial x_{j}}=2 \mu \frac{\partial \varepsilon_{i j}}{\partial x_{j}}=\mu \frac{\partial^{2} u_{i}}{\partial x_{j} \partial x_{j}}$.

\subsection{Convective and pressure gradient projections}

The computation of the residual terms are simplified if we introduce the convective and pressure gradient projections $c_{i}$ and $\pi_{i}$, respectively, defined as

$$
\begin{aligned}
c_{i} & =r_{m_{i}}-\rho u_{j} \frac{\partial u_{i}}{\partial x_{j}} \\
\pi_{i} & =r_{m_{i}}-\frac{\partial p}{\partial x_{i}} .
\end{aligned}
$$

We can express $r_{m_{i}}$ in Eqs. (16a) and (16b) in terms of $c_{i}$ and $\pi_{i}$, respectively which then become additional variables. The system of integral equations is now augmented in the necessary number of equations by imposing that the residual $r_{m_{i}}$ vanishes (in average sense) for both forms given by Eqs. (18). This gives the final system of governing equations as:

$$
\begin{aligned}
\int_{\Omega} & {\left[\delta u_{i} \rho\left(\frac{\partial u_{i}}{\partial t}+u_{j} \frac{\partial u_{i}}{\partial x_{j}}\right)+\frac{\partial \delta u_{i}}{\partial x_{j}}\left(\mu \frac{\partial u_{i}}{\partial x_{j}}-\delta_{i j} p\right)\right] \mathrm{d} \Omega } \\
& -\int_{\Omega} \delta u_{i} b_{i} \mathrm{~d} \Omega-\int_{\Gamma_{t}} \delta u_{i} t_{i} \mathrm{~d} \Gamma
\end{aligned}
$$



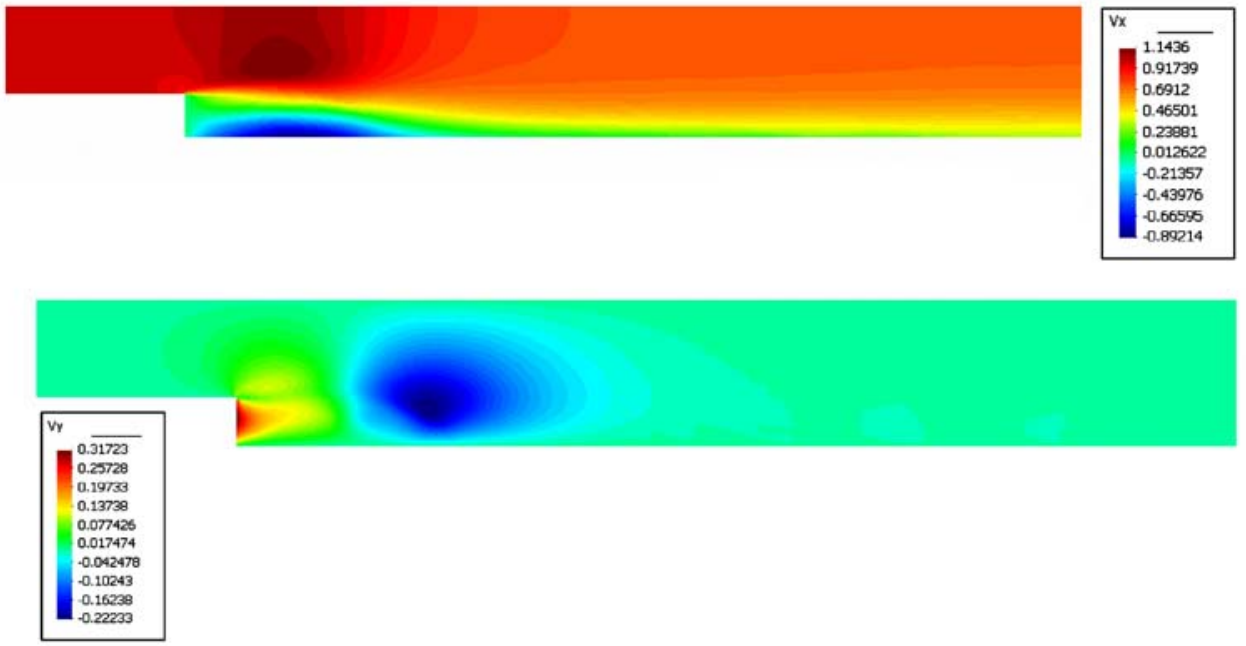

Fig. 4 Backward facing step. Contours of horizontal (above) and vertical velocities

$$
\begin{gathered}
+\int_{\Omega} \frac{h_{i k}}{2} \frac{\partial\left(\delta u_{i}\right)}{\partial x_{k}}\left(\rho u_{j} \frac{\partial u_{i}}{\partial x_{j}}+c_{i}\right) \mathrm{d} \Omega=0 \\
\int_{\Omega} q \frac{\partial u_{i}}{\partial x_{i}} \mathrm{~d} \Omega+\int_{\Omega} \sum_{i=1}^{n_{d}} \tau_{i} \frac{\partial q}{\partial x_{i}}\left(\frac{\partial p}{\partial x_{i}}+\pi_{i}\right) \mathrm{d} \Omega=0 \\
\int_{\Omega} \delta c_{i} \rho\left(\rho u_{j} \frac{\partial u_{i}}{\partial x_{j}}+c_{i}\right) \mathrm{d} \Omega=0 \text { no sum in } i \\
\int_{\Omega} \delta \pi_{i} \tau_{i}\left(\frac{\partial p}{\partial x_{i}}+\pi_{i}\right) \mathrm{d} \Omega=0 \text { no sum in } i
\end{gathered}
$$

with $i, j, k=1, n_{d}$. In Eqs. (21) and (22) $\delta c_{i}$ and $\delta \pi_{i}$ are appropriate weighting functions and the $\rho$ and $\tau_{i}$ weights are introduced for convenience.

We note that accounting for the convective and pressure gradient projections enforces the consistency of the formulation as it ensures that the stabilization terms in Eqs. (19) and (20) have a residual form which vanishes for the "exact" solution. Neglecting these terms can reduce the accuracy of the numerical solution and it makes the formulation more sensitive to the value of the stabilization parameters as shown in references [51-53].

\section{Finite element discretization}

We choose $C^{\circ}$ continuous linear interpolations of the velocities, the pressure, the convection projections $c_{i}$ and the pressure gradient projections $\pi_{i}$ over 3 -noded triangles (2D) and 4-noded tetrahedra (3D). The linear interpolations are written as

$$
\begin{aligned}
& u_{i}=N^{k} \bar{u}_{i}^{k}, \quad p=N^{k} \bar{p}^{k} \\
& c_{i}=N^{k} \bar{c}_{i}^{k}, \quad \pi_{i}=N^{k} \bar{\pi}_{i}^{k},
\end{aligned}
$$

where the sum goes over the number of nodes of each element $n$ ( $n=3 / 4$ for triangles/tetrahedra), $(\cdot)^{k}$ denotes the nodal variables and $N^{k}$ are the linear shape functions [1].

Substituting the approximations (23) into Eqs. (19)-(22) and choosing the Galerking form with $\delta u_{i}=q=\delta c_{i}=$ $\delta \pi_{i}=N^{i}$ leads to following system of discretized equations

$$
\begin{aligned}
& \mathbf{M} \dot{\overline{\mathbf{u}}}+\mathbf{H} \overline{\mathbf{u}}-\mathbf{G} \overline{\mathbf{p}}+\mathbf{C} \overline{\mathbf{c}}=\mathbf{f} \\
& \mathbf{G}^{\mathrm{T}} \overline{\mathbf{u}}+\hat{\mathbf{L}} \overline{\mathbf{p}}+\mathbf{Q} \overline{\boldsymbol{\pi}}=\mathbf{0} \\
& \hat{\mathbf{C}} \overline{\mathbf{u}}+\mathbf{M} \overline{\mathbf{c}}=\mathbf{0} \\
& \mathbf{Q}^{\mathrm{T}} \overline{\mathbf{p}}+\hat{\mathbf{M}} \overline{\boldsymbol{\pi}}=\mathbf{0},
\end{aligned}
$$

where

$\mathbf{H}=\mathbf{A}+\mathbf{K}+\hat{\mathbf{K}}$.

If we denote the node indexes with superscripts $a, b$, the space indices with subscripts $i, j$, the element contributions to the components of the arrays involved in these equations are $(i, j=1,3$ for $3 \mathrm{D}$ problems)

$M_{i j}^{a b}=\left(\int_{\Omega^{e}} \rho N^{a} N^{b} \mathrm{~d} \Omega\right) \delta_{i j}, \quad A_{i j}^{a b}=\left(\int_{\Omega^{e}} \rho N^{a}\left(\mathbf{u}^{\mathrm{T}} \nabla N^{b}\right) \mathrm{d} \Omega\right) \delta_{i j}$

$K_{i j}^{a b}=\left(\int_{\Omega^{e}} \mu \nabla^{\mathrm{T}} N^{a} \nabla N^{b} \mathrm{~d} \Omega\right) \delta_{i j}, \quad \nabla=\left[\frac{\partial}{\partial x_{1}}, \frac{\partial}{\partial x_{2}}, \frac{\partial}{\partial x_{3}}\right]^{\mathrm{T}}$

$\hat{K}_{i j}^{a b}=\left(\frac{1}{2} \int_{\Omega^{e}} h_{i j} \frac{\partial N^{a}}{\partial x_{j}}\left(\rho \mathbf{u}^{\mathrm{T}} \nabla N^{b}\right) \mathrm{d} \Omega\right) \delta_{i j}, \quad G_{i}^{a b}=\int_{\Omega^{e}} \frac{\partial N^{a}}{\partial x_{i}} N^{b} \mathrm{~d} \Omega$

$\mathbf{C}=\left[\begin{array}{l}\mathbf{C}_{1} \\ \mathbf{C}_{2} \\ \mathbf{C}_{3}\end{array}\right], \quad C_{i}^{a b}=\frac{1}{2} \int_{\Omega^{e}} h_{i j} \frac{\partial N^{a}}{\partial x_{j}} N^{b} \mathrm{~d} \Omega$

$\hat{L}^{a b}=\int_{\Omega^{e}}\left(\nabla^{\mathrm{T}} N^{a}\right)[\tau] \nabla N^{b} \mathrm{~d} \Omega, \quad[\tau]=\left[\begin{array}{ccc}\tau_{1} & 0 & 0 \\ 0 & \tau_{2} & 0 \\ 0 & 0 & \tau_{3}\end{array}\right]$

$\mathbf{Q}=\left[\mathbf{Q}_{1}, \mathbf{Q}_{2}, \mathbf{Q}_{3}\right], \quad Q_{i}^{a b}=\int_{\Omega^{e}} \tau_{i} \frac{\partial N^{a}}{\partial x_{i}} N^{b} \mathrm{~d} \Omega \quad$ no sum in $i$ 


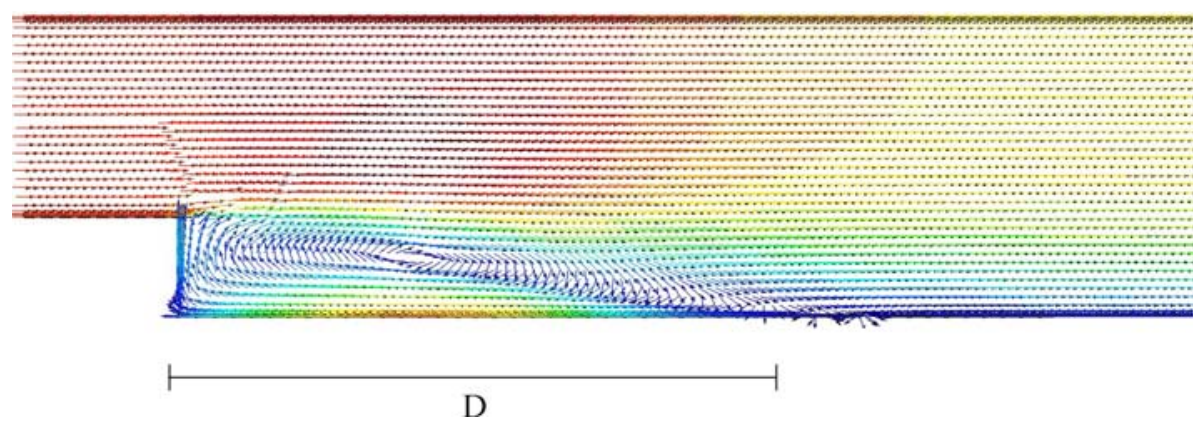

Fig. 5 Backward facing step. Velocity vectors and recirculation distance $D$

$\hat{\mathbf{C}}=\left[\hat{\mathbf{C}}_{1}, \hat{\mathbf{C}}_{2}, \hat{\mathbf{C}}_{3}\right], \quad \hat{C}_{1}^{a b}=\hat{C}_{2}^{a b}=\hat{C}_{3}^{a b}=\int_{\Omega^{e}} \rho^{2} N^{a}\left(\mathbf{u}^{\mathrm{T}} \nabla N^{b}\right) \mathrm{d} \Omega$

$\hat{M}_{i j}^{a b}=\left(\int_{\Omega^{e}} \tau_{i} N^{a} N^{b} \mathrm{~d} \Omega\right) \delta_{i j}, \quad f_{i}^{a}=\int_{\Omega^{e}} N^{a} f_{i} \mathrm{~d} \Omega+\int_{\Gamma^{e}} N^{a} t_{i} \mathrm{~d} \Gamma$.

It is understood that all the arrays are matrices (except $\mathbf{f}$ which is a vector) whose components are obtained by grouping together the left indices in the previous expressions ( $a$ and possibly $i$ ) and the right indices ( $b$ and possibly $j$ ).

Note that the stabilization matrix $\hat{\mathbf{K}}$ in Eq. (25) adds additional orthotropic diffusivity terms of value $\rho\left(h_{i j} u_{l} / 2\right)$.

The overall stabilization terms introduced by the FIC formulation above presented have the intrinsic capacity to ensure physically sound numerical solutions for a wide spectrum of Reynolds numbers without the need of introducing additional turbulence modelling terms. This interesting property is validated in the solution of the examples presented in a next section.

\subsection{Transient solution scheme}

The solution in time of the system of Eqs. (24) can be written in general form as

$$
\begin{aligned}
& \mathbf{M} \frac{1}{\Delta t}\left(\overline{\mathbf{u}}^{n+1}-\overline{\mathbf{u}}^{n}\right)+\mathbf{H}^{n+\theta} \overline{\mathbf{u}}^{n+\theta}-\mathbf{G} \overline{\mathbf{p}}^{n+\theta} \\
& \quad+\mathbf{C}^{n+\theta} \overline{\mathbf{c}}^{n+\theta}=\mathbf{f}^{n+\theta} \\
& \mathbf{G}^{\mathrm{T}} \overline{\mathbf{u}}^{n+\theta}+\hat{\mathbf{L}}^{n+\theta} \overline{\mathbf{p}}^{n+\theta}+\mathbf{Q} \overline{\boldsymbol{\pi}}^{n+\theta}=\mathbf{0} \\
& \hat{\mathbf{C}}^{n+\theta} \overline{\mathbf{u}}^{n+\theta}+\mathbf{M} \overline{\mathbf{c}}^{n+\theta}=\mathbf{0} \\
& \mathbf{G}^{\mathrm{T}} \overline{\mathbf{p}}^{n+\theta}+\hat{\mathbf{M}}^{n+\theta} \overline{\boldsymbol{\pi}}^{n+\theta}=\mathbf{0}
\end{aligned}
$$

where $\mathbf{H}^{n+\theta}=\mathbf{H}\left(\mathbf{u}^{n+\theta}\right)$, etc and the parameter $\theta \in[0,1]$. The direct monolithic solution of Eqs. (27) is possible using an adequate iterative scheme $[52,53]$. However, in our work we have used the fractional step method described next.

\section{Fractional step method}

A fractional step scheme is derived by spliting the discretized momentum equation (27a) into the two following equations

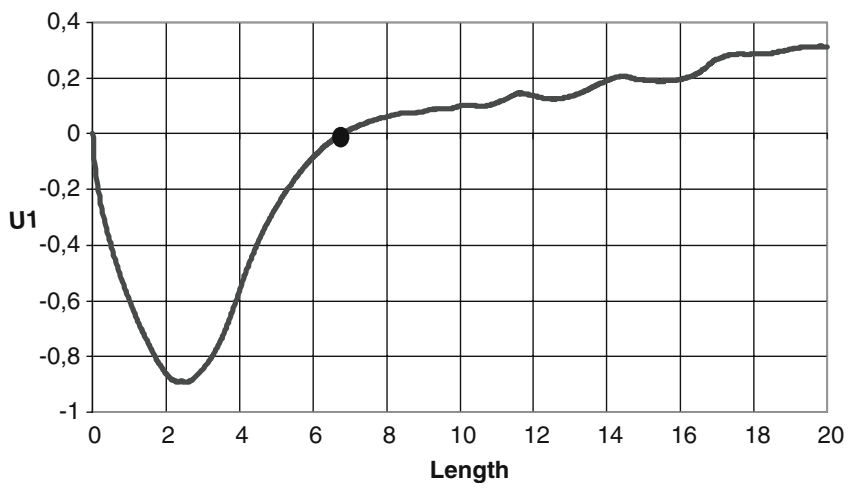

Fig. 6 Distribution of the horizontal velocity along the bottom line starting from the vertical wall of the step. The circle shows the end of the recirculation region.

$$
\begin{aligned}
& \mathbf{M} \frac{1}{\Delta t}\left(\tilde{\mathbf{u}}^{n+1}-\overline{\mathbf{u}}^{n}\right)+\mathbf{H}^{n+\theta} \tilde{\mathbf{u}}^{n+\theta}-\alpha \mathbf{G} \overline{\mathbf{p}}^{n} \\
& \quad+\mathbf{C}^{n+\theta} \overline{\mathbf{c}}^{n+\theta}=\mathbf{f}^{n+\theta} \\
& \mathbf{M} \frac{1}{\Delta t}\left(\overline{\mathbf{u}}^{n+1}-\tilde{\mathbf{u}}^{n+1}\right)-\mathbf{G}\left(\overline{\mathbf{p}}^{n+1}-\alpha \overline{\mathbf{p}}^{n}\right)=\mathbf{0} .
\end{aligned}
$$

In Eqs. (28) $\tilde{\mathbf{u}}^{n+1}$ is a predicted value of the velocity at time $n+1$ and $\alpha$ is a variable whose values of interest are zero and one. For $\alpha=0$ (first order scheme) the splitting error is of order $0(\Delta t)$, whereas for $\alpha=1$ (second order scheme) the error is of order $0\left(\Delta t^{2}\right)$ [52]. We have chosen $\alpha=1$ for the solution of the examples presented in the paper.

Equations (28) are completed with the following three equations emanating from Eqs. (27b-d)

$\mathbf{G}^{\mathrm{T}} \overline{\mathbf{u}}^{n+1}+\hat{\mathbf{L}}^{n} \overline{\mathbf{p}}^{n+1}+\mathbf{Q} \overline{\boldsymbol{\pi}}^{n}=\mathbf{0}$

$\hat{\mathbf{C}}^{n+1} \overline{\mathbf{u}}^{n+1}+\mathbf{M} \overline{\mathbf{c}}^{n+1}=\mathbf{0}$

$\mathbf{Q}^{\mathrm{T}} \overline{\mathbf{p}}^{n+1}+\hat{\mathbf{M}}^{n+1} \overline{\boldsymbol{\pi}}^{n+1}=\mathbf{0}$.

The value of $\overline{\mathbf{u}}^{n+1}$ obtained from Eq. (28b) is substituted into Eq. (29a) to give

$$
\begin{aligned}
& \mathbf{G}^{\mathrm{T}} \tilde{\mathbf{u}}^{n+1}+\Delta t \mathbf{G}^{\mathrm{T}} \mathbf{M}^{-1} \mathbf{G}\left(\overline{\mathbf{p}}^{n+1}-\alpha \overline{\mathbf{p}}^{n}\right) \\
& \quad+\hat{\mathbf{L}}^{n} \mathbf{p}^{n+1}+\mathbf{Q} \overline{\boldsymbol{\pi}}^{n}=\mathbf{0} .
\end{aligned}
$$

The product $\mathbf{G}^{\mathrm{T}} \mathbf{M}^{-1} \mathbf{G}$ can be approximated by a laplacian matrix, i.e.

$\mathbf{G}^{\mathrm{T}} \mathbf{M}^{-1} \mathbf{G}=\frac{1}{\rho} \mathbf{L} \quad$ with $L^{a b}=\int_{\Omega^{e}} \nabla^{\mathrm{T}} N^{a} \nabla N^{b} \mathrm{~d} \Omega$, 


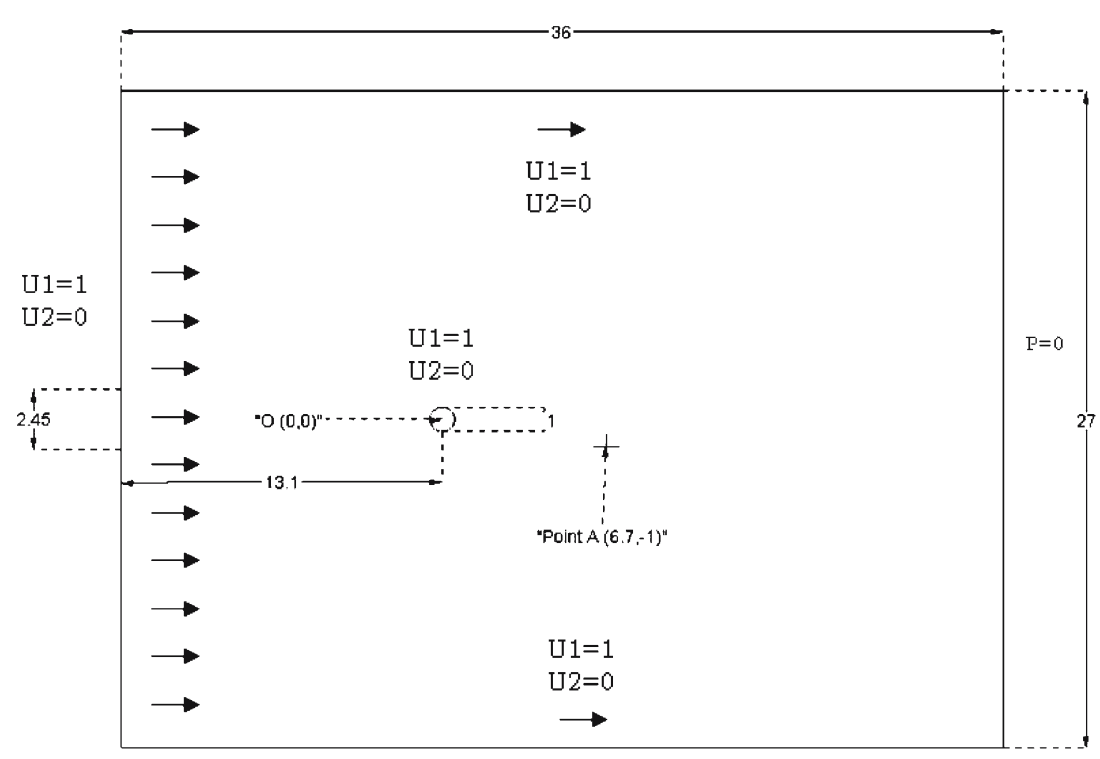

Fig. 7 Flow past a cylinder of unit diameter. Analysis domain and boundary conditions

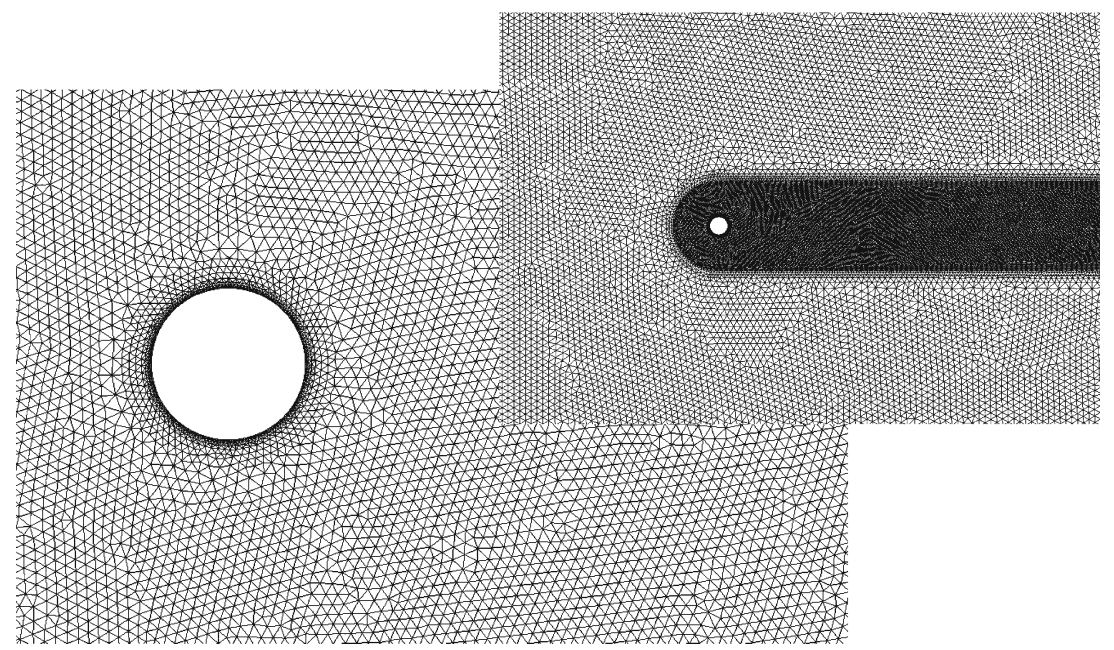

Fig. 8 Flow past a cylinder. Mesh of 91,316 three-noded triangles used for the computations

where $L^{a b}$ are the element contributions to $\mathbf{L}$.

The steps of the fractional step scheme are:

Step 1 Eq.(28a) is linearized as

$\mathbf{M} \frac{\tilde{\mathbf{u}}^{n+1}-\overline{\mathbf{u}}^{n}}{\Delta t}+\tilde{\mathbf{H}}^{n+\theta} \tilde{\mathbf{u}}^{n+\theta}-\alpha \mathbf{G} \overline{\mathbf{p}}^{n}+\tilde{\mathbf{C}}^{n+\theta} \overline{\mathbf{c}}^{n}=\overline{\mathbf{f}}^{n+\theta}$,

where $\tilde{\mathbf{u}}^{n+\theta}=\theta \tilde{\mathbf{u}}^{n+1}+(1-\theta) \overline{\mathbf{u}}^{n}, \tilde{\mathbf{H}}^{n+\theta}=\mathbf{H}\left(\tilde{\mathbf{u}}^{n+\theta}\right)$, and $\tilde{\mathbf{C}}^{n+\theta}=\mathbf{C}\left(\tilde{\mathbf{u}}^{n+\theta}\right)$. We have chosen in our computation $\theta=0$. For this value, the fractional nodal velocities $\tilde{\mathbf{u}}^{n+1}$ can be explicitely computed from Eq. (32) by

$\tilde{\mathbf{u}}^{n+1}=\overline{\mathbf{u}}^{n}-\Delta t \mathbf{M}_{d}^{-1}\left[\tilde{\mathbf{H}}^{n} \overline{\mathbf{u}}^{n}-\alpha \mathbf{G} \overline{\mathbf{p}}^{n}+\tilde{\mathbf{C}}^{n} \overline{\mathbf{c}}^{n}-\overline{\mathbf{f}}^{n}\right]$,

where $\mathbf{M}_{d}$ is the lumped diagonal form of $\mathbf{M}$.
Step 2 Compute $\overline{\mathbf{p}}^{n+1}$ from Eq. (30) as

$\overline{\mathbf{p}}^{n+1}=-\left[\hat{\mathbf{L}}^{n}+\frac{\Delta t}{\rho} \mathbf{L}\right]^{-1}\left[\mathbf{G}^{\mathrm{T}} \tilde{\mathbf{u}}^{n+1}-\alpha \frac{\Delta t}{\rho} \mathbf{L} \overline{\mathbf{p}}^{n}+\mathbf{Q} \overline{\boldsymbol{\pi}}^{n}\right]$.

Step 3 Compute $\overline{\mathbf{u}}^{n+1}$ explicitly from Eq. (28a) as

$\overline{\mathbf{u}}^{n+1}=\tilde{\mathbf{u}}^{n+1}+\Delta t \mathbf{M}_{d}^{-1} \mathbf{G}\left(\overline{\mathbf{p}}^{n+1}-\alpha \overline{\mathbf{p}}^{n}\right)$.

Step 4 Compute $\overline{\mathbf{c}}^{n+1}$ explicitly from Eq. (29b) as

$\overline{\mathbf{c}}^{n+1}=-\mathbf{M}_{d}^{-1} \hat{\mathbf{C}}^{n+1} \overline{\mathbf{u}}^{n+1}$.

Step 5 Compute $\overline{\boldsymbol{\pi}}^{n+1}$ explicitly from Eq. (29c) as

$\overline{\boldsymbol{\pi}}^{n+1}=-\hat{\mathbf{M}}_{d}^{-1} \mathbf{Q}^{\mathrm{T}} \overline{\mathbf{p}}^{n+1}$. 


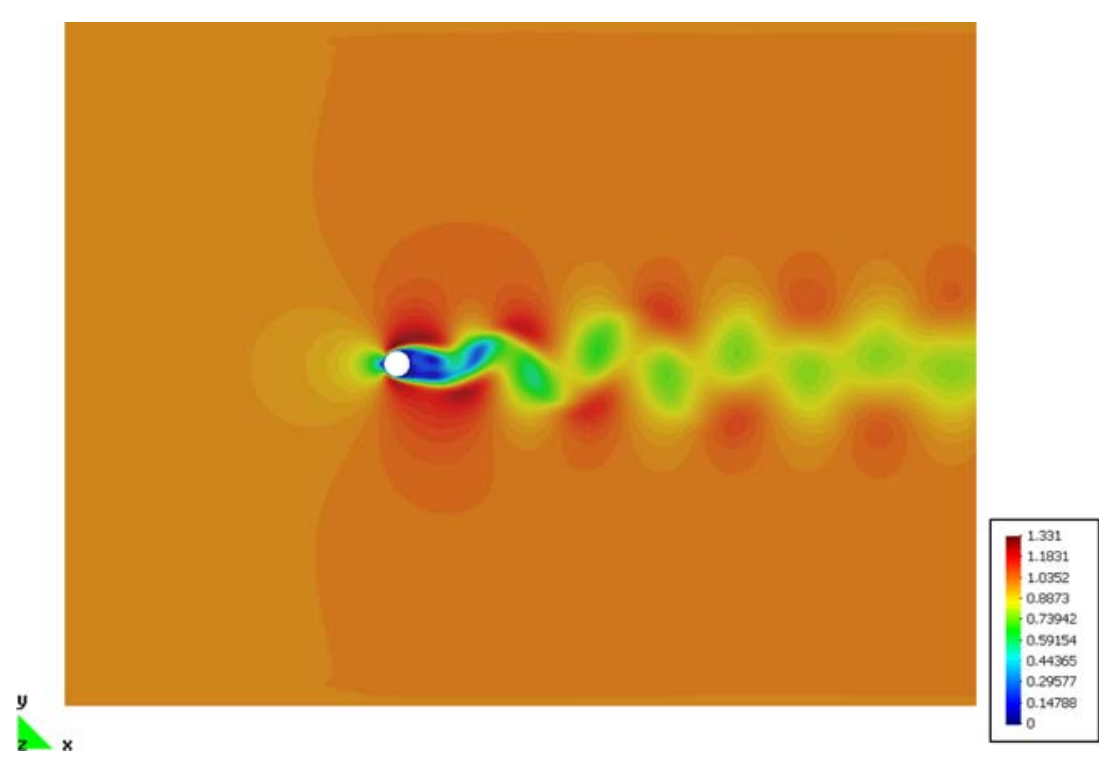

Fig. 9 Flow past a cylinder, $R e=100$. Contour of the velocity vector modulus for $t=100 \mathrm{~s}$

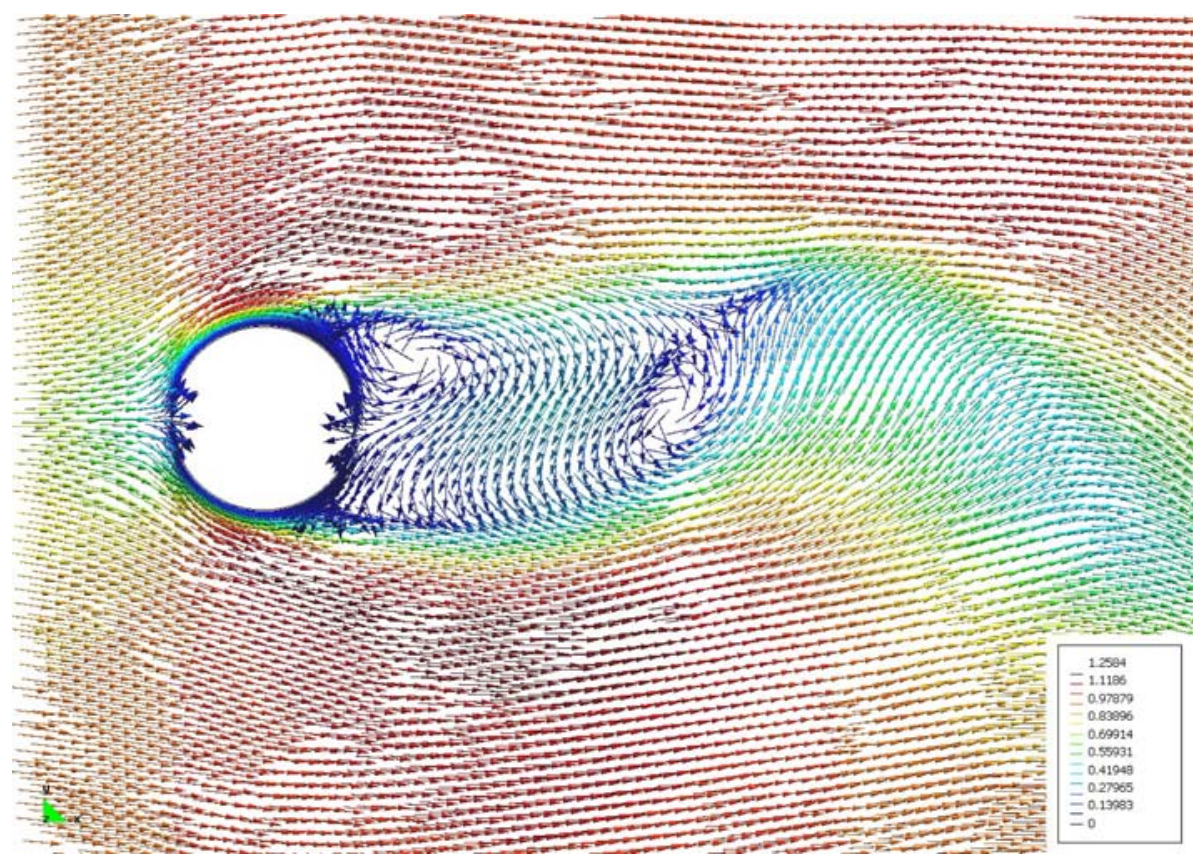

Fig. 10 Flow past a cylinder, $R e=100$. Velocity vectors for $t=100 \mathrm{~s}$

Above algorithm has improved stabilization properties versus the standard segregation methods due to the introduction of the laplacian matrix $\hat{\mathbf{L}}$ in Eq. (34) which emanates from the FIC stabilization terms.

The boundary conditions are applied as follows. No condition is applied in the computation of the fractional velocities $\tilde{\mathbf{u}}^{n+1}$ in Eq. (33). The prescribed velocities at the boundary are applied when solving for $\overline{\mathbf{u}}^{n+1}$ in the step 3. The prescribed pressures at the boundary are imposed by making $\overline{\mathbf{p}}^{n}$ equal to the prescribed pressure values.

\section{Stokes flow}

The formulation for a Stokes flow can be readily obtained simply by neglecting the convective terms in the general $\mathrm{Na}$ vier-Stokes formulation. Consequently, the convective stabilization terms and the convective projection variables are not larger necessary. Also the intrinsic time parameters $\tau_{i}$ take now the simpler form [see Eq. (12)]:

$\tau_{i}=\frac{3 h_{i i} h_{i}}{8 \mu}$. 

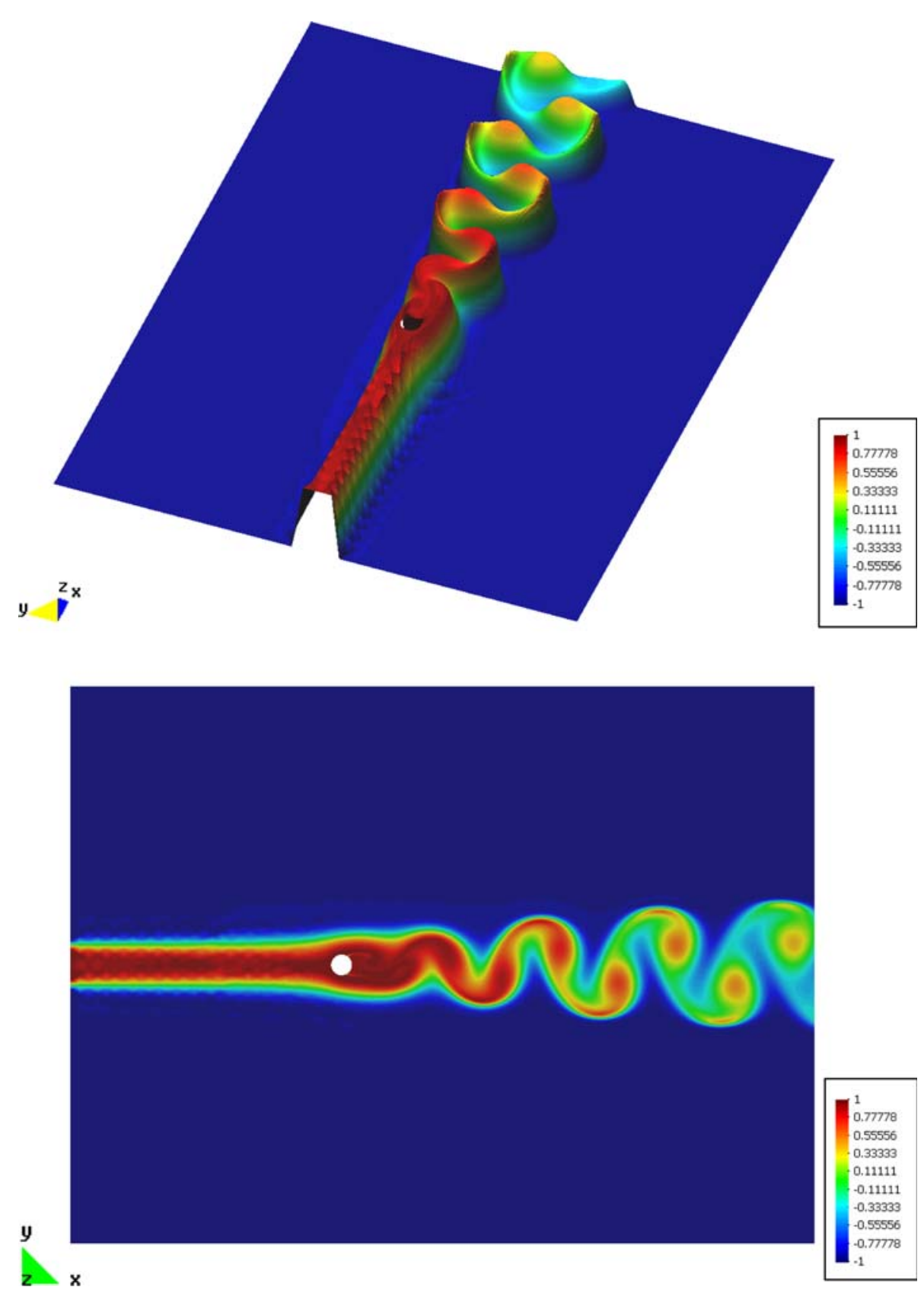

Fig. 11 Flow past a cylinder, $R e=100$. Trajectories of a substance over a band of $2.45 \mathrm{U}$ at the entry transported across the flow for $t=100 \mathrm{~s}$

The resulting discretized system of equations can be written as [see Eqs. (29)]

$$
\begin{aligned}
& \mathbf{M} \dot{\overline{\mathbf{u}}}+\mathbf{K} \overline{\mathbf{u}}-\mathbf{G} \overline{\mathbf{p}}=\mathbf{f} \\
& \mathbf{G}^{\mathrm{T}} \overline{\mathbf{u}}+\hat{\mathbf{L}} \overline{\mathbf{p}}+\mathbf{Q} \overline{\boldsymbol{\pi}}=\mathbf{0} \\
& \mathbf{Q}^{\mathrm{T}} \overline{\mathbf{p}}+\hat{\mathbf{M}} \overline{\boldsymbol{\pi}}=\mathbf{0} .
\end{aligned}
$$

The fractional step algorithm of the previous section can now be implemented. We note that convergence of the predictor-corrector scheme is now faster due to the absence of the non linear convective terms in the momentum equation.
The steady-state form of Eqs. (39) can be expressed in matrix form as

$$
\left[\begin{array}{ccc}
\mathbf{K} & -\mathbf{G} & \mathbf{0} \\
-\mathbf{G}^{\mathrm{T}} & -\hat{\mathbf{L}} & -\mathbf{Q} \\
\mathbf{0} & -\mathbf{Q}^{\mathrm{T}} & -\hat{\mathbf{M}}
\end{array}\right]\left\{\begin{array}{c}
\overline{\mathbf{u}} \\
\overline{\mathbf{p}} \\
\overline{\bar{\pi}}
\end{array}\right\}=\left\{\begin{array}{l}
\mathbf{f} \\
\mathbf{0} \\
\mathbf{0}
\end{array}\right\} .
$$

The system is symmetric and always positive definite and therefore leads to a non singular solution. This property holds for any interpolation function chosen for $\overline{\mathbf{u}}, \overline{\mathbf{p}}$ and $\overline{\boldsymbol{\pi}}$, therefore overcoming the Babuška-Brezzi (BB) restrictions [1].

A reduced velocity-pressure formulation can be obtained by eliminating the pressure gradient projection variables $\overline{\boldsymbol{\pi}}$ 


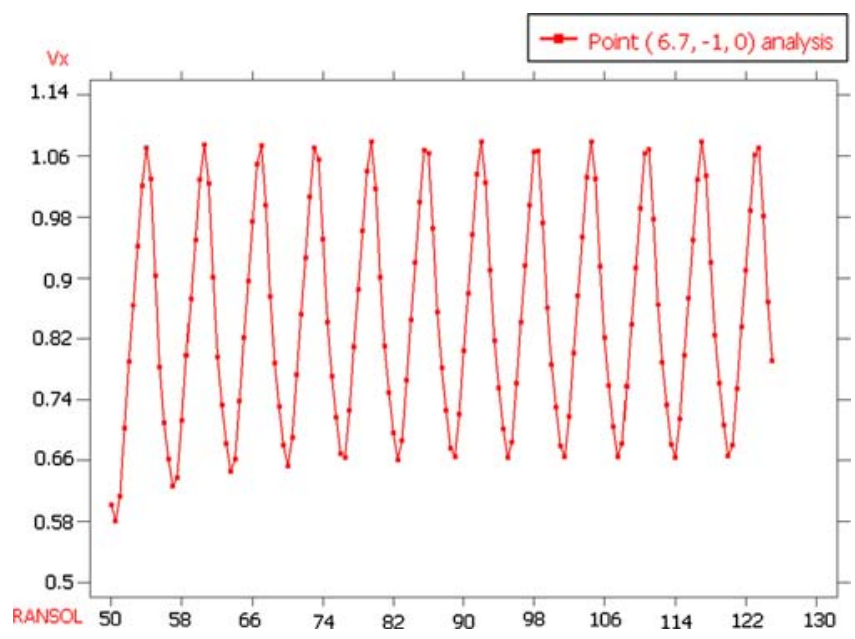

Fig. 12 Flow past a cylinder, $R e=100$. Oscillations with time of the horizontal velocity at the point with coordinates $A(6.7-1.02)$

from the last equation to give

$\left[\begin{array}{cc}\mathbf{K} & -\mathbf{G} \\ -\mathbf{G}^{\mathrm{T}}-\left(\hat{\mathbf{L}}-\mathbf{Q} \hat{\mathbf{M}}^{-1} \mathbf{Q}^{\mathrm{T}}\right)\end{array}\right]\left\{\begin{array}{l}\overline{\mathbf{u}} \\ \overline{\mathbf{p}}\end{array}\right\}=\left\{\begin{array}{l}\mathbf{f} \\ \mathbf{0}\end{array}\right\}$.

The reduction process is simplified by using a diagonal form of matrix $\hat{\mathbf{M}}$. Applications of this scheme to incompressible solid mechanics problems are reported in $[52,53]$.

\section{Computation of the characteristic distances}

The computation of the stabilization parameters is a crucial issue as they affect both the stability and accuracy of the numerical solution. The different procedures to compute the stabilization parameters are typically based on the study of simplified forms of the stabilized equations. Contributions to this topic are reported in [11-20, 26-31, 34, 45, 54, 55]. Despite the relevance of the problem there still lacks a general method to compute the stabilization parameters for all the range of flow situations.

Recent work of the authors in the application of the FIC/ FEM formulation to convection-diffusion problems with sharp arbitrary gradients $[39,41]$ has shown that the stabilizing FIC terms take the form of a simple orthotropic diffusion if the balance equation is written in the principal curvature directions of the solution. Excellent results were reported in [39,41] by computing first the characteristic length distances along the principal curvature directions, followed by a standard transformation of the distances to global axes. The resulting stabilized finite element equations capture the high gradient zones in the vicinity of the domain edges (boundary layers) as well as the sharp gradients appearing randomly in the interior of the domain $[39,41]$. The FIC/FEM thus reproduces the best features of both the so called transverse (cross-wind) dissipation or shock capturing methods [56, 57].
The numerical computations are simplified without apparent loss of accuracy if the main principal curvature direction of the solution at each element point is approximated by the direction of the gradient vector at the element center. The second principal direction (for 2D problems) is taken in the orthogonal direction to the gradient. For linear triangles and quadrilaterals these directions are assumed to be constant within the element $[39,41]$.

Above simple scheme has been extended in this work for the computation of the characteristic distances $h_{i j}$ for the momentum equations. As for the length parameters $h_{i}$ in the mass conservation equation, the simplest assumption $h_{i}=h_{i i}$ has been taken. Details of the algorithm for computing $h_{i j}$ are given next (the method is explained for 2D problems although it is readily extendible to $3 \mathrm{D}$ problems).

For the $i$-th momentum balance equation and every step of the fractional step method described in Sect. 4:

1. A coordinate system $\vec{\xi}_{1}^{i}, \vec{\xi}_{2}^{i}$ is defined at each element point such that $\vec{\xi}_{1}^{i}$ is aligned with the gradient of $u_{i}\left(\vec{\xi}_{1}^{i}=\overrightarrow{\nabla u_{i}}\right)$ and $\vec{\xi}_{2}^{i}$ is orthogonal to $\vec{\xi}_{1}^{i}$ in anticlockwise sense (Fig. 1). The angle that $\vec{\xi}_{1}^{i}$ forms with the global $x_{1}$ axis is defined as $\alpha_{i}$. Recall that the upper and lower index $i$ denotes the $i$ th momentum equation.

2. The element characteristic distances $l_{i 1}$ and $l_{i 2}$ are defined as the maximum projections of the element sides along the $\vec{\xi}_{1}^{i}$ and $\vec{\xi}_{2}^{i}$ axes, respectively (Fig. 2).

3. The characteristic distances $h_{i 1}$ and $h_{i 2}$ are computed as

$$
\left\{\begin{array}{l}
h_{i 1} \\
h_{i 2}
\end{array}\right\}=\left[\begin{array}{cc}
c_{i} & -s_{i} \\
s_{i} & c_{i}
\end{array}\right]\left\{\begin{array}{l}
\bar{h}_{i 1} \\
\bar{h}_{i 2}
\end{array}\right\}, \quad i=1,2
$$

with $c_{i}=\cos \alpha_{i}, s_{i}=\sin \alpha_{i}$ and the local distances $\bar{h}_{i 1}$ and $\bar{h}_{i 2}$ are

$$
\bar{h}_{i 1}=\left(\operatorname{coth} \bar{\gamma}_{i j}-\frac{1}{\bar{\gamma}_{i j}}\right) l_{i j}, \quad \bar{\gamma}_{i j}=\frac{\bar{u}_{j} l_{i j}}{2 \mu} j=1,2,
$$

where $\bar{u}_{1}$ and $\bar{u}_{2}$ are the components of the velocity vector along the local axes $\vec{\xi}_{1}^{i}$ and $\vec{\xi}_{2}^{i}$, respectively (Fig. 1).

\section{Examples}

The examples were solved with the Tdyn code where the formulation here presented has been implemented. The Tdyn code can be downloaded from the webpage given in [58].

\subsection{Backward facing step at high Reynolds number}

Figure 3 shows the geometry of the standard backward facing step problem. The boundary conditions were the following: $u_{1}=1$ and $u_{2}=0$ were taken at the entry while $p=0$ was assumed at the exit. Slipping conditions were assumed 


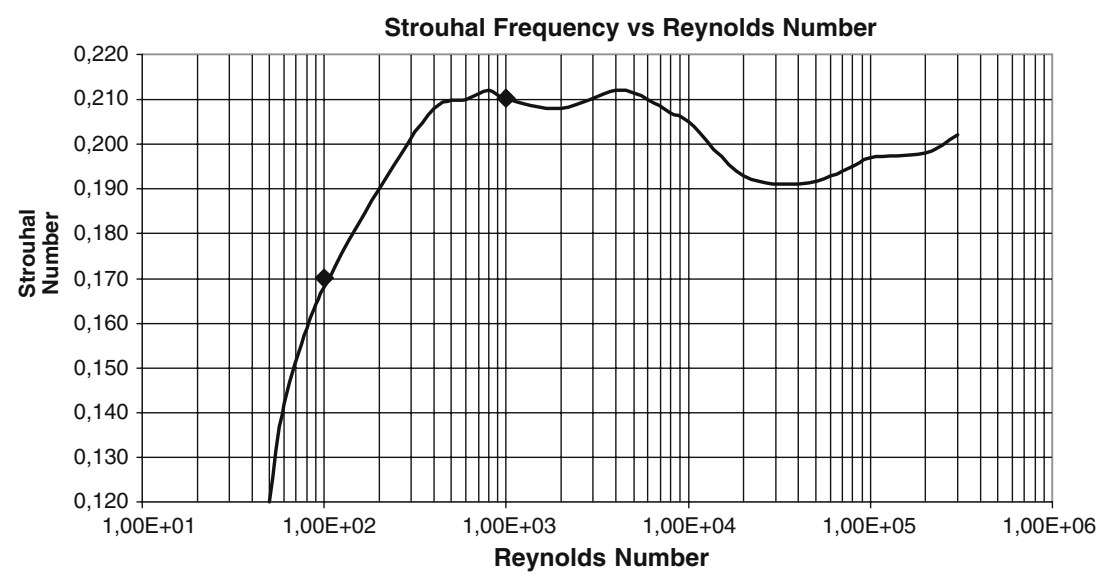

Fig. 13 Flow past a cylinder. Experimental (thick line) and computed (filled diamond) values of the Strouhal number $S$ in terms of the Reynolds number. Experimental values taken from [http://wn7.enseeiht.fr/hmf/travaux/CD0102/travaux/optmfn/gpfmho/01- 02/grp1/index.htm]

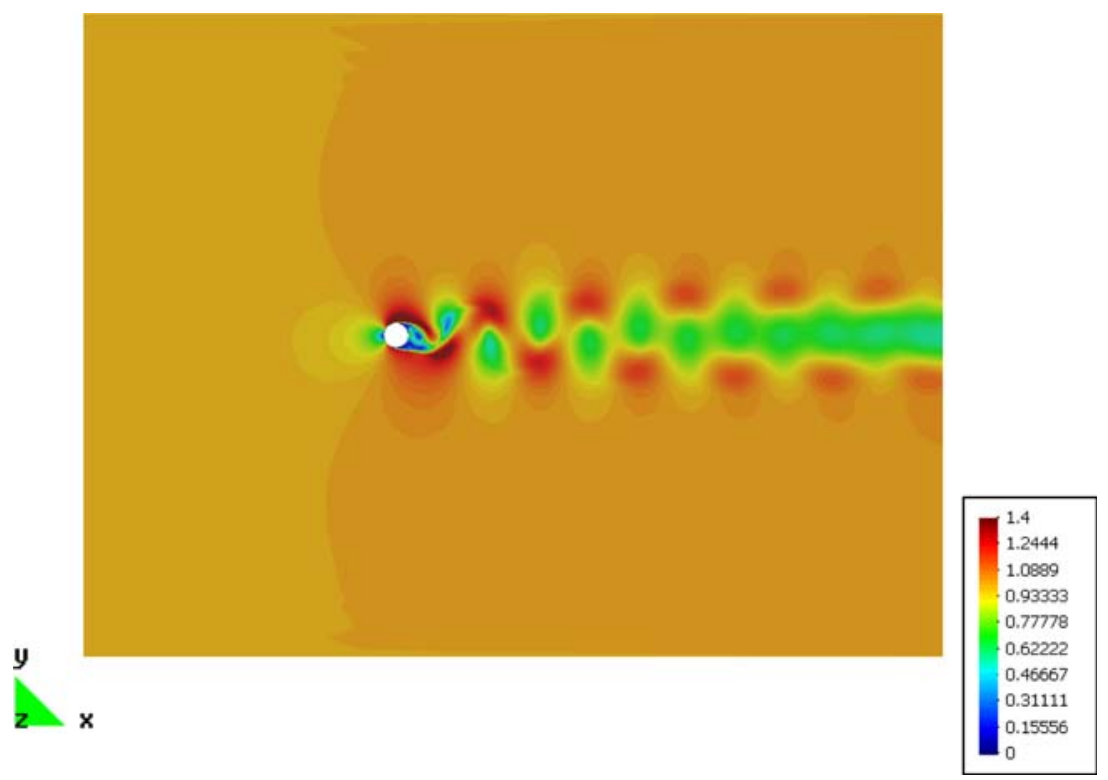

Fig. 14 Flow past a cylinder, $R e=1,000$. Contour of the velocity vector module for $t=100 \mathrm{~s}$

at the rest of the vertical and horizontal walls. A value of the kinematic viscosity $v=(\mu / \rho)=2.1 \times 10^{-5}$ was taken giving a Reynolds number of $R e=\left(\left(u_{\text {free }} H\right) / \nu\right)=47,619$ for $H=1$ and $u_{\text {free }}=1$.

Figure 3 also shows the relatively coarse mesh chosen of 30,850 three-noded triangular elements and 15,426 nodes. The contours of the horizontal and vertical velocities and details of the velocity vectors are shown in Figs. 4 and 5, respectively. Figure 6 shows the distribution of the horizontal velocity along the bottom line starting from the vertical wall of the step. The point where the horizontal velocity changes sign indicates the end of the recirculation area.

The length of the circulation area computed from Fig. 6 is 6.79. This value compares very well $(3.2 \%)$ with the experimental value reported by Kim et al. [59] (see Table 1). The FIC/FEM results are remarkably accurate in comparison with
Table 1 Backward facing step. Length of the recirculation distance $D$ for $R e=47,619$

\begin{tabular}{llll}
\hline $\begin{array}{l}\text { Model } \\
\text { Exp. [59] }\end{array}$ & $\begin{array}{l}\text { Length } D \\
6.0-7.0\end{array}$ & Error range (\%) & Average error (\%) \\
\hline K- $\varepsilon[60]$ & 5.2 & $13.3-26$ & 19.6 \\
K- $\varepsilon[61]$ & 5.88 & $2-16$ & 9 \\
K- $\varepsilon$ [62] & 6.0 & $0-14$ & 7 \\
K- $\varepsilon$ [63] & 6.2 & $13.7-11.4$ & 12.6 \\
K-Tau [63] & 6.82 & $13.7-2.5$ & 8.1 \\
FIC/FEM & 6.71 & $11.8-4.1$ & 7.9 \\
\hline
\end{tabular}

Comparison of the FIC/FEM result with experimental data and with numerical results obtained using different turbulence models

other results reported in the literature obtained using $K-\varepsilon$ and $K$-tau turbulence models [60-63]. We note again that the FIC/FEM formulation does not include any additional turbulence terms. 


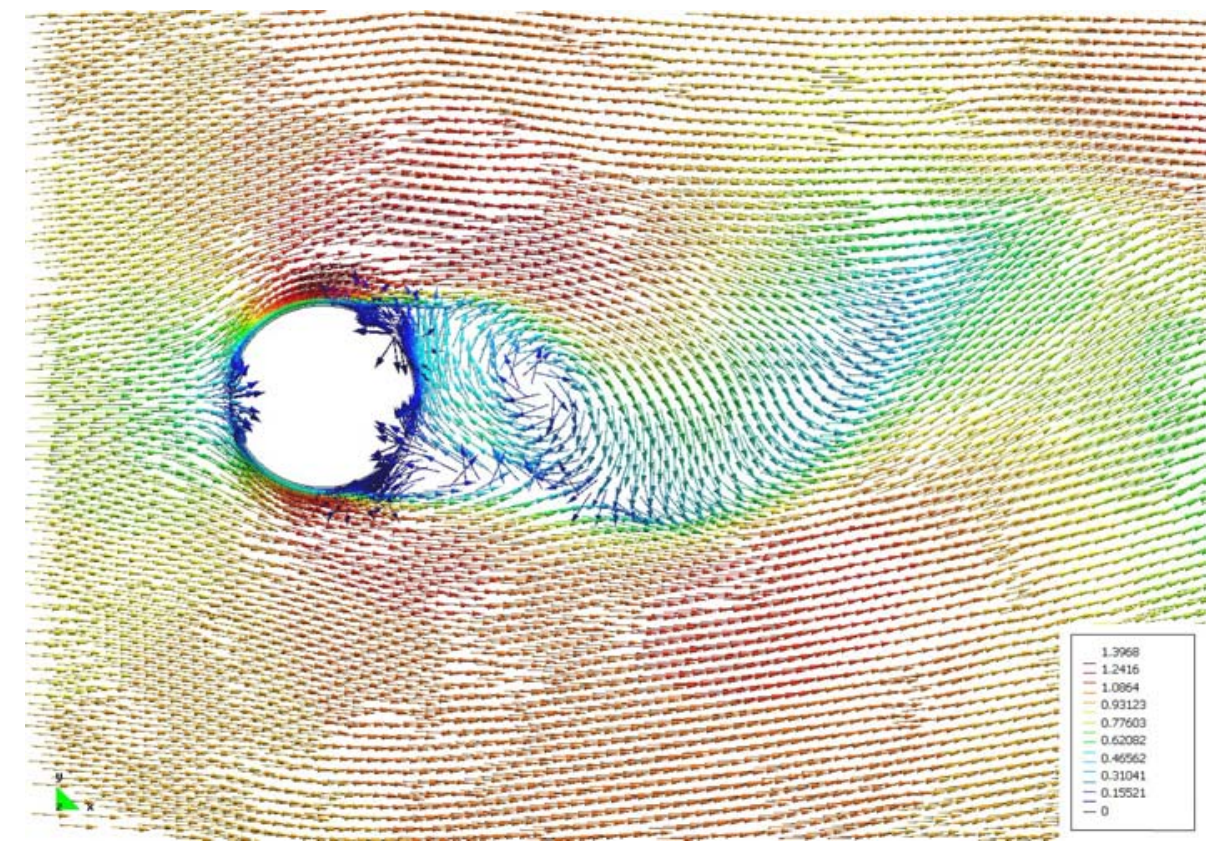

Fig. 15 Flow past a cylinder, $R e=1,000$. Velocity vectors for $t=100 \mathrm{~s}$

7.2 Flow past a cylinder. Computation of the Strouhal instability

Figure 7 shows the geometry for the analysis of the flow past a cylinder of unit diameter $(D)$. A unit horizontal velocity is prescribed at the inlet boundary and at the two horizontal walls. Zero pressure is prescribed at the outlet boundary. The dimensions of the analysis domain are $36 \times 27$ units. The origin of the coordinate system has been sampled at the center of the cylinder located at a distance of 13.1 units from the entry wall. Zero velocity is prescribed at the cylinder wall. The kinematic viscosity is $v=0.01$. Figure 8 shows the mesh of 91,316 three-noded elements used for the computation. A detail of the mesh in the vicinity of the cylinder is also shown in Fig. 8.

The problem has been analyzed first for a value of the horizontal velocity at the entry of $u_{1}=1$ giving a Reynolds number of $R e=100$. Figures 9 and 10, respectively, show the velocity modulus contours and the velocity vectors for $t=100 \mathrm{~s}$.

Figure 11 shows images of the trajectory of a substance over a band of 2.45 units transported at the entry across the flow for $t=100 \mathrm{~s}$. The picture shows clearly the oscillatory nature of the flow.

Figure 12 shows the oscillations of the horizontal velocity at the point $A$ with coordinates $(6.7,-1.02)$ with time. The Strouhal number computed from the shedding frequency $n$ as $S=(n D /|\mathbf{u}|)$ is $S=0.1702$. This number compares very well with the experimental result available in the literature (see Fig. 13).

The same problem was analyzed for a value of the kinematic viscosity $v=0.001$ giving $R e=1,000$. The same mesh of 91,316 linear triangles of Fig. 7 was used.
Figures 14, 15, 16 show, respectively, the velocity modulus contours, the velocity vectors in the vicinity of the cylinder for $t=100 \mathrm{~s}$. and the trajectories of a substance transported across the flow. Figure 17 finally shows the oscillations of the horizontal velocity at point $A$. The computed value of the Strouhal number in this case was $S=0.2103$. This value again coincides well with the reported experimental data (see Fig. 13).

It is a well known fact that for $R e>300$ the flow past a cylinder exhibits 3D features. In [64] results from $2 \mathrm{D}$ and $3 \mathrm{D}$ computation were compared for $R e=300$ and 800 . While 3D features were observed even at $R e=300$ and more so at $R e=800$, there were no large discrepances between the global flow parameters (such as drag, lift and Strouhal number) obtained from 2D and 3D computations. These conclusions justify the results of the 2D computations presented in the paper.

\section{Conclusions}

The finite calculus (FIC) form of the fluid mechanics equations is a good starting point for deriving stabilized FEM for solving a variety of incompressible fluid flow problems. The matrix stabilization terms introduced by the FIC formulation here presented allow to obtain physically sound solutions in the presence of sharp gradients occuring for high Reynolds numbers without the need of introducing a turbulence model. Good numerical solutions have been obtained in the 2D examples solved with relatively coarse meshes for moderate and high values of the Reynolds number. These preliminary results reinforce our idea that the stabilization terms introduced by the FIC formulation suffice to provide 

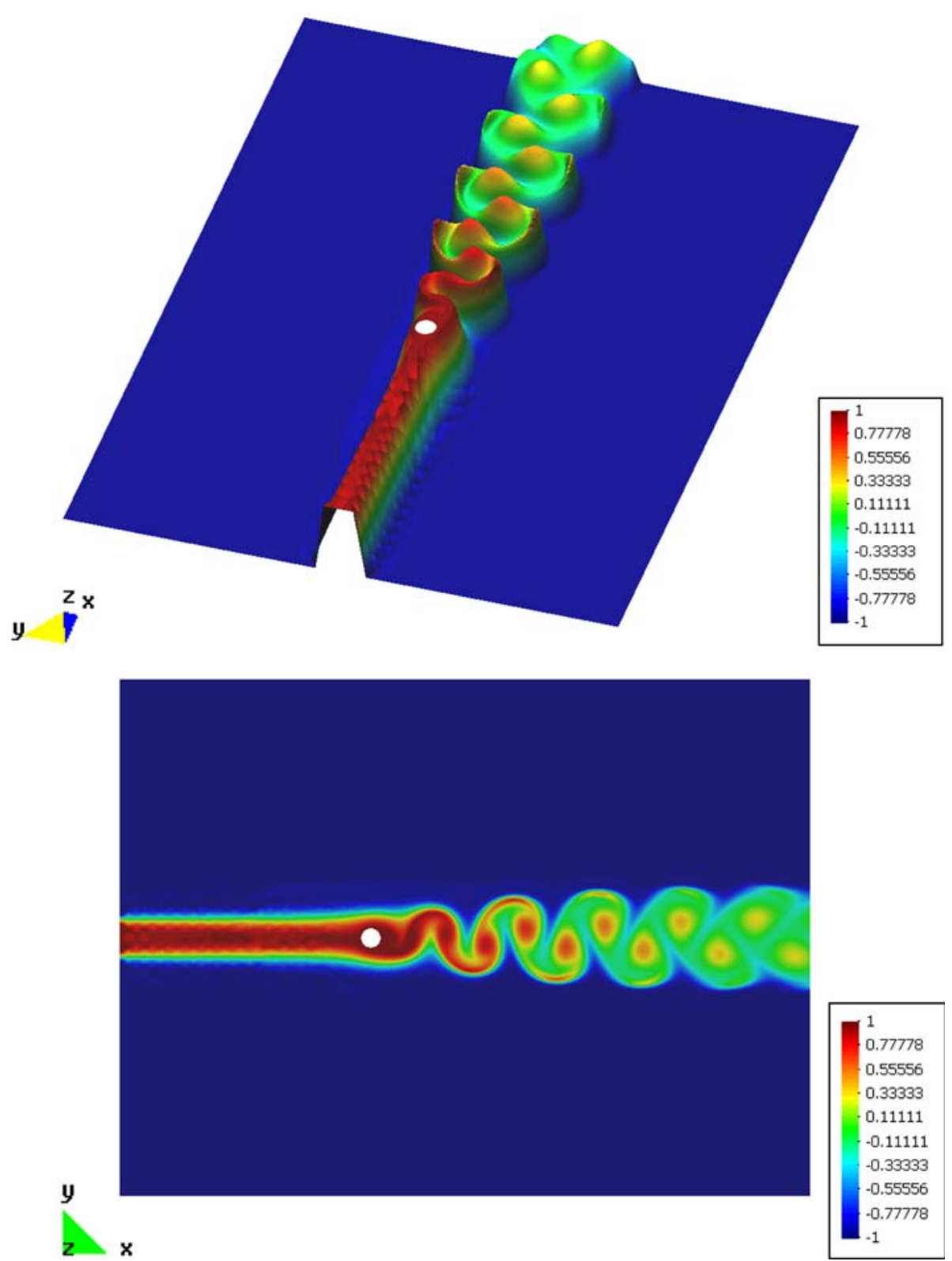

Fig. 16 Flow past a cylinder, $R e=1,000$. Trajectories of a substance over a band of 2.45 units at the entry transported across the flow for $t=100 \mathrm{~s}$

good results for problems for which turbulence models are required using alternative numerical methods. These results also confirm the close link between the stabilized methods and turbulence models, which surely will be the object of much research in the near future.

\section{Appendix}

The FIC momentum equations in two dimensions (2D) are obtained by expressing the balance of momentum along the horizontal and vertical directions in the finite domains shown in Figs. 18 and 19, respectively.
The balance equation is written for each finite domain as

$$
\sum f_{i} \mathrm{~d} \Omega=\frac{\partial}{\partial t} \int_{\Omega} \rho u_{i} \mathrm{~d} \Omega+\int_{\Gamma}\left(\rho u_{i}\right) \mathbf{u}^{\mathrm{T}} \mathbf{n} \mathrm{d} \Gamma \quad i=1,2,
$$

where $f_{i}$ includes the forces due to the stresses acting on the boundary of the balance domain and the body forces per unit area (Figs. 18, 19).

Expressing the values of the momentum and force terms at the end point of the balance domain in terms the values at an arbitrary point (such as the corner point A) using higher order Taylor expansions and retaining second order tems gives after 


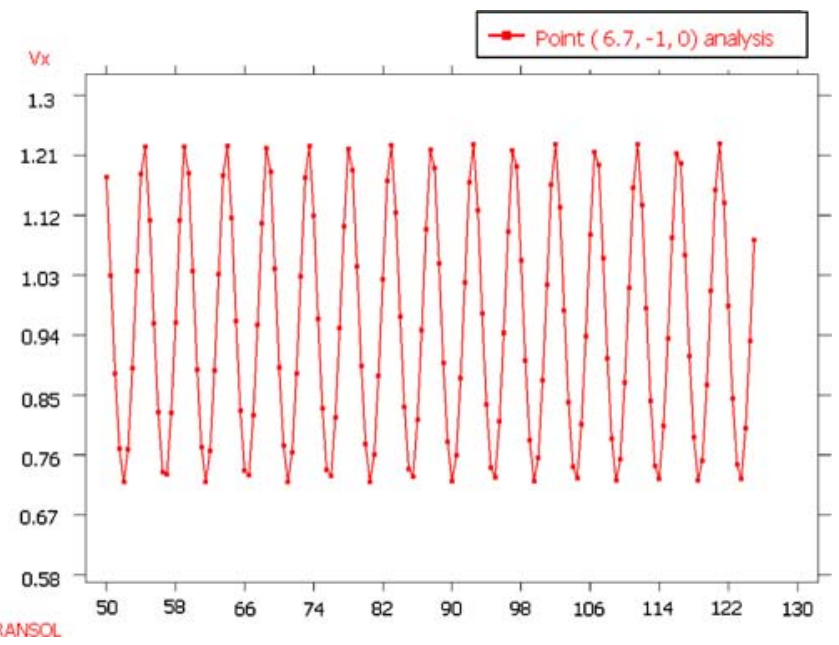

Fig. 17 Flow past a cylinder, $R e=1,000$. Oscillations with time of the horizontal velocity at the point with coordinates $A(6.7-1.02)$
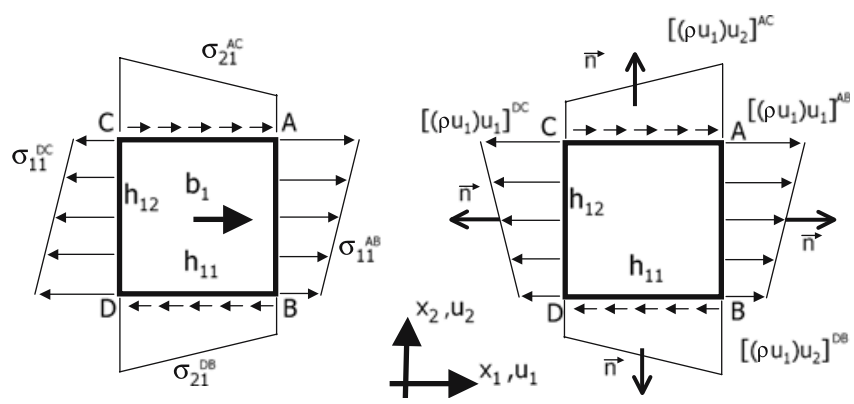

Fig. 18 Finite domain where balance of momentum is imposed along the horizontal direction

some algebra [32] the FIC momentum equations along the $i$ th coordinate direction as

$r_{i}-\frac{1}{2} h_{i j} \frac{\partial r_{i}}{\partial x_{j}}=0 \quad i, j=1,2$

with

$r_{i}:=\rho\left(\frac{\partial u_{i}}{\partial t}+u_{j} \frac{\partial u_{i}}{\partial x_{j}}\right)-\frac{\partial \sigma_{i j}}{\partial x_{j}}-b_{i}$

with $\sigma_{i j}=s_{i j}-p \delta_{i j}$ where $s_{i j}$ and $p$ are the deviatoric stresses and the pressure, respectively.

Note that distance $h_{12}$ is arbitrary when writting the balance of momentum along the $x_{1}$ direction. The same applies for the distance $h_{21}$ when deriving the balance equation along the $x_{2}$ direction. Thus, in general, $h_{12} \neq h_{21}$ and this explains the matrix form of the FIC momentum equations.

The FIC mass balance equation is obtained by invoking the balance of mass in the finite domain of Fig. 20

$\int_{\Gamma} \rho \mathbf{u}^{\mathrm{T}} \mathbf{n} \mathrm{d} \Gamma=0$

Expanding the values of $\rho u_{i}$ at the corner points in terms of the value at an arbitrary point gives of the mass balance

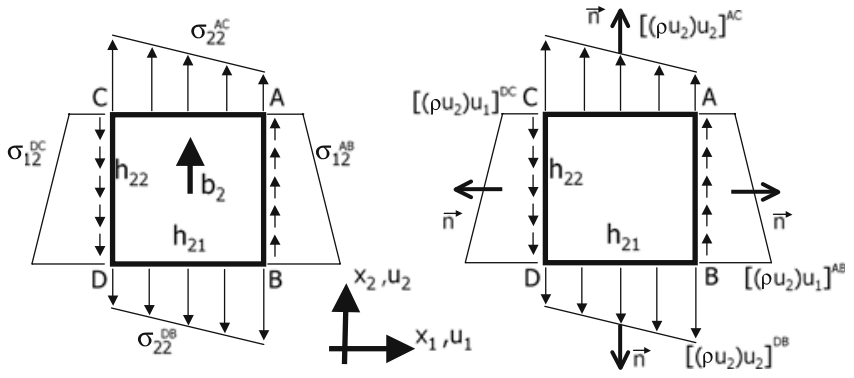

Fig. 19 Finite domain where balance of momentum is imposed along the vertical direction

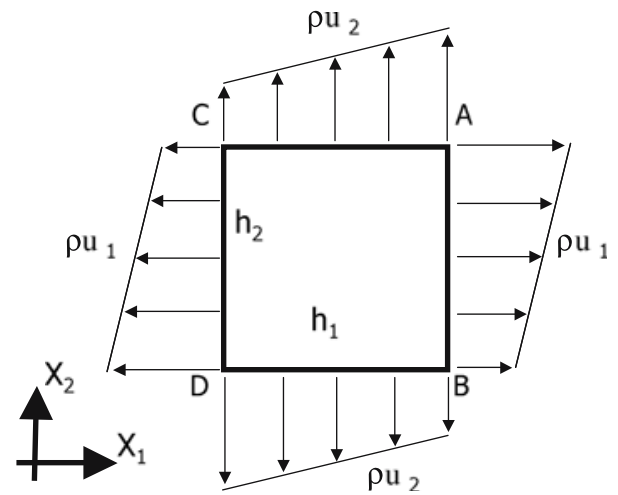

Fig. 20 Finite domain where balance of mass is enforced

domain the FIC mass balance equation as [32, 43]

$\frac{\partial u_{i}}{\partial x_{i}}-\frac{1}{2} h_{j} \frac{\partial}{\partial x_{j}}\left(\frac{\partial u_{i}}{\partial x_{i}}\right)=0$.

Note that a matrix form of the characteristic distances is not obtained in this case as the mass balance equation expresses the conservation of the mass in the whole domain ABCD of Fig. 20 with dimensions $h_{1}$ and $h_{2}$. Distances $h_{1}$ and $h_{2}$ should be taken in general different from distances $h_{i j}$ defining the domain where balance of momentum is enforced. In our computations we have however assumed that $h_{1}=h_{11}$ and $h_{2}=h_{22}$ for simplicity.

Acknowledgements The authors thank Prof. S.R. Idelsohn and C.A. Felippa for many useful discussions.

\section{References}

1. Zienkiewicz OC, Taylor RC, Nietharasu P (2005) The finite element method, 6th edn, Vol 3. Elsevier, Amsterdam

2. Kelly DW, Nakazawa S, Zienkiewicz OC (1980) A note on anisotropic balancing dissipation in the finite element method approximation to convective diffusion problems. Int J Numer Methods Eng 15:1705-1711

3. Briley WR, Neerarambam SS, Whitfield DL (1995) Multigrid algorithm for three-dimensional incompressible high-Reynolds number turbulent flows. AIAA J 33(1):2073-2079

4. Peraire J, Morgan K, Peiro J (1994) The simulation of 3D incompressible flows using unstructured grids. In: Caughey DA, Hafez MM (eds) Frontiers of computational fluid dynamics, Chapter 16, Wiley, New York 
5. Sheng C, Taylor LK, Whitfield DL (1996) Implicit lower-upper/ approximate-factorization schemes for incompressible flows. J Comput Phys 128(1):32-42

6. Storti M, Nigro N, Idelsohn SR (1995) Steady state incompressible flows using explicit schemes with an optimal local preconditioning. Comput Methods Appl Mech Eng 124:231-252

7. Hirsch C (1990) Numerical computation of internal and external flow, vol. 1 1988, vol. 2. Wiley, New York

8. Heinrich JC, Hayakorn PS, Zienkiewicz OC (1977) An upwind finite element scheme for two dimensional convective transport equations. Int J Numer Methods Eng 11:131-143

9. Idelsohn SR, Storti M, Nigro N (1995) Stability analysis of mixed finite element formulation with special mention to stabilized equalorder interpolations. Int J Numer Methods Fluids 20:1003-1022

10. Idelsohn SR, Nigro N, Storti M, Buscaglia G (1996) A Petrov-Galerkin formulation for advection-reaction-diffusion. Comput Methods Appl Mech Eng 136:27-46

11. Brooks A, Hughes TJR (1982) Streamline upwind/Petrov-Galerkin formulation for convection dominated flows with particular emphasis on the incompressible Navier-Stokes equations. Comput Methods Appl Mech Eng 32:199-259

12. Hughes TJR, Tezduyar TE (1984) Finite element methods for first-order hyperbolic systems with particular emphasis on the compressible Euler equations. Comput Methods Appl Mech Eng $45 \cdot 217-284$

13. Tezduyar TE, Park YJ (1986) Discontinuity capturing finite element formulations for nonlinear convection-diffusion-reaction equations. Comput Methods Appl Mech Eng 59:307-325

14. Hughes TJR, Mallet M (1986) A new finite element formulations for computational fluid dynamics: III. The generalized streamline operator for multidimensional advective-diffusive systems. Comput Methods Appl Mech Eng 58:305-328

15. Hughes TJR, Franca LP, Balestra M (1986) A new finite element formulation for computational fluid dynamics. V Circumventing the Babuska-Brezzi condition: A stable Petrov-Galerkin formulation of the Stokes problem accomodating equal order interpolations. Comput Methods Appl Mech Eng 59:85-99

16. Franca LP, Frey SL (1992) Stabilized finite element methods: II. The incompressible Navier-Stokes equations. Comput Methods Appl Mech Eng 99:209-233

17. Hughes TJR, Hauke G, Jansen K (1994) Stabilized finite element methods in fluids: Inspirations, origins, status and recent developments. In: Recent Developments in Finite Element Analysis. A Book Dedicated to Taylor RL, Hughes TJR, Oñate E, Zienkiewicz OC (eds) (International center for numerical methods in engineering, Barcelona, Spain, pp 272-292

18. Cruchaga MA, Oñate E (1997) A finite element formulation for incompressible flow problems using a generalized streamline operator. Comput Methods Appl Mech Eng 143:49-67

19. Cruchaga MA, Oñate E (1999) A generalized streamline finite element approach for the analysis of incompressible flow problems including moving surfaces. Comput Methods Appl Mech Eng 173:241-255

20. Hughes TJR, Franca LP, Hulbert GM (1989) A new finite element formulation for computational fluid dynamics: VIII. The Galerkin/least-squares method for advective-diffusive equations. Comput Methods Appl Mech Eng 73:173-189

21. Tezduyar TE, Mittal S, Ray SE, Shih R (1992) Incompressible flow computations with stabilized bilinear and linear equal order interpolation velocity-pressure elements. Comput Methods Appl Mech Eng 95:221-242

22. Donea J (1984) A Taylor-Galerkin method for convective transport problems. Int J Numer Methods Eng 20:101-119

23. Pironneau O (1982) On the transport-diffusion algorithm and its applications to the Navier-Stokes equations. Numer Math 38:309

24. Löhner R, Morgan K, Zienkiewicz OC (1984) The solution of nonlinear hyperbolic equation systems by the finite element method. Int J Numer Methods Fluids 4:1043

25. Codina R, Vazquez M, Zienkiewicz OC (1998) A general algorithm for compressible and incompressible flow - Part III. The semi-implicit form. Int J Numer Methods Fluids 27:13-32
26. Codina R, Zienkiewicz OC (2002) CBS versus GLS stabilization of the incompressible Navier-Stokes equations and the role of the time step as stabilization parameter. Commun Numer Methods Eng 18(2):99-112

27. Codina R, Blasco J (2000) Stabilized finite element method for the transient Navier-Stokes equations based on a pressure gradient operator. Comput Methods Appl Mech Eng 182:277-301

28. Hughes TJR (1995) Multiscale phenomena: green functions, subgrid scale models, bubbles and the origins of stabilized methods. Comput Methods Appl Mech Eng 127:387-401

29. Brezzi F, Franca LP, Hughes TJR, Russo A (1997) $b=\int g$. Comput Methods Appl Mech Eng 145:329-339

30. Codina R (2002) Stabilized finite element approximation of transient incompressible flows using orthogonal subscales. Comput Methods Appl Mech Eng 191:4295-4321

31. Donea J, Huerta A (2003) Finite element method for flow problems. Wiley, New York

32. Oñate E (1998) Derivation of stabilized equations for advectivediffusive transport and fluid flow problems. Comput Methods Appl Mech Eng 151:233-267

33. Oñate E (2004) Possibilities of finite calculus in computational mechanics. Int J Numer Methods Eng 60(1):255-281

34. Oñate E, García J, Idelsohn S (1997) Computation of the stabilization parameter for the finite element solution of advective-diffusive problems. Int J Numer Methods Fluids 25:1385-1407

35. Oñate E, García J, Idelsohn S (1998) An alpha-adaptive approach for stabilized finite element solution of advective-diffusive problems with sharp gradients. Ladeveze P, Oden JT (eds) New Adv. in Adaptive Comp. Met. in Mech. Elsevier, Amsterdam

36. Oñate E, Manzan M (1999) A general procedure for deriving stabilized space-time finite element methods for advective-diffusive problems. Int J Numer Methods Fluids 31:203-221

37. Oñate E, Manzan M (2000) Stabilization techniques for finite element analysis of convection diffusion problems. In: Comini G, Sunden B (eds) Computational analysis of heat transfer. WIT Press, Southampton

38. Oñate E, Idelsohn S (1998) A mesh free finite point method for advective-diffusive transport and fluid flow problems. Comput Mech 21:283-292

39. Oñate E, Zárate F, Idelsohn SR (2006) Finite element formulation for convective-diffusive problems with sharp gradients using finite calculus. Comput Methods Appl Mech Eng 195:1793-1825

40. Oñate E, Miquel J, Hauke G (2005) Stabilized formulation for the advection-diffusion-absorption equation using finite calculus and linear finite elements. Comput Methods Appl Mech Eng

41. Oñate E, Miquel J, Zárate F (2005) Stabilized solution of the multidimensional advection-diffusion-absorption equation using linear finite elements. Comput Methods Appl Mech Eng

42. Felippa CA, Oñate E (2005) Nodally exact Ritz discretizations of 1D diffusion-absorption and Helmholtz equations by variational FIC and modified equation methods. Comput Mech (in press)

43. Oñate $E$ (2000) A stabilized finite element method for incompressible viscous flows using a finite increment calculus formulation. Comput Methods Appl Mech Eng 182:1-2, 355-370

44. Oñate E, Sacco C, Idelsohn SR (2000) A finite point method for incompressible flow problems. Computing and visualization in science 2:67-75

45. Oñate E, García J (2001) A finite element method for fluid-structure interaction with surface waves using a finite calculus formulation. Comput Methods Appl Mech Eng 191:635-660

46. García J, Oñate E (2003) An unstructured finite element solver for ship hydrodynamic problems. J Appl Mech 70:18-26

47. Idelsohn SR, Oñate E, Del Pin F (2003) A lagrangian meshless finite element method applied to fluid-structure interaction problems. Comput Struct 81:655-671

48. Oñate E, Idelsohn SR, Del Pin F, Aubry R (2004) The particle finite element method. An overview. Int J Comput Methods 1(2):267307

49. Idelsohn SR, Oñate E, Del Pin F (2004) The Particle Finite Element Method: a powerful tool to solve incompressible flows with freesurfaces and breaking waves. Int J Numer Methods Eng 61:964989 
50. Oñate E, García J, Idelsohn SR (2004) Ship hydrodynamics. In: Stein E, de Borst R, Hughes TJR (eds) Encyclopedia of computational mechanics, vol 3, Chapter 18. Wiley, New York, pp 579-607

51. Oñate E, García J, Idelsohn SR, Del Pin F (2006) FIC formulations for finite element analysis of incompressible flows. Eulerian, ALE and Lagrangian approaches. Comput Methods Appl Mech Eng

52. Oñate E, Rojek J, Taylor RL, Zienkiewicz OC (2004) Finite calculus formulation for incompressible solids using linear triangles and tetrahedra. Int J Numer Methods Eng 59:1473-1500

53. Oñate E, Taylor RL, Zienkiewicz OC, Rojek J (2003) A residual correction method based on finite calculus. Eng Comput 20(5/6):629-658

54. Tezduyar TE, Osawa Y (2000) Finite element stabilization parameters computed from element matrices and vectors. Comput Methods Appl Mech Eng 190:411-430

55. Tezduyar TE (2003) Computation of moving boundaries and interfaces and stabilization parameters. Int J Numer Methods Fluids 43:555-575

56. Hughes TJR, Mallet M (1986) A new finite element formulations for computational fluid dynamics: IV. A discontinuity capturing operator for multidimensional advective-diffusive system. Comput Methods Appl Mech Eng 58:329-336
57. Codina R (1993) A discontinuity-capturing crosswind dissipation for the finite element solution of the convection-diffusion equation. Comput Methods Appl Mech Eng 110:325-342

58. Tdyn (2005) A finite element code for fluid-dynamic analysis. COMPASS Ingeniería y Sistemas SA. www.compassis.com

59. Kim JJ (1978) Investigation of separation and reattachment of turbulent shear layer: flow over a backward facing step. PhD Thesis, Standford Univeristy

60. Mansour NN, Morel T (1981) In: AFOSR-HTTM Stanford conference on complex turbulent flows III. Stanford University, CA, pp 1418-1423

61. Pollard A (1981) In: AFOSR-HTTM Stanford conference on complex turbulent flows III. Stanford University, CA, pp 1486-1490

62. Abdelmengui AM, Goh SY, Ilegbusi J, Spalding DB (1981) Predictions of complex turbulent flows using the PHOENICS computer code. In: AFOSR-HTTM Stanford conference on complex turbulent flows III. Stanford University, CA, pp 1521-1526

63. Illinca F, Hetu J-F, Pelletier D (1998) A unified finite element algorithm for two-equations models of turbulence. Comput Fluids 27(3):291-310

64. Kalro V, Tezduyar T (1997) Parallel 3D computation of unsteady flows around circular cylinders. Parallel Comput 23:1235-1248 